\title{
Quantum Core Affect: Process-semantic theory of emotions
}

\author{
Ilya A. Surov \\ ITMO University, Saint-Petersburg, Russia
}

\begin{abstract}
The paper describes model of human affect based on quantum theory of semantics. The model considers emotion as subjective representation of behavioral context relative to a basis binary choice, organized by cyclical process structure and an orthogonal evaluation axis. The resulting spherical space, generalizing well-known circumplex models, accommodates basic emotions in specific angular domains. Predicted process-semantic structure of affect is observed in the word2vec data, as well as in the previously obtained spaces of emotion concepts. The established quantumtheoretic structure of affective space connects emotion science with quantum models of cognition and behavior, opening perspective for synergetic progress in these fields.
\end{abstract}

\section{KEYWORDS}

core affect, emotion, semantics, process cycle, quantum cognition, qubit

\section{Introduction}

Scientific conquer of a novel terrain starts from outlining the scope of target phenomena, identifying basic observation techniques, and definition of fundamental terms. In the case of emotion, immensity of relevant phenomenology and measurements (involving most of human cognition, communication and language, large parts of biology, neurophysiology, and behavior) has no parallel in "hard" part of natural sciences. Third of the above steps is therefore outstandingly complex, so that ordering of phenomenological and conceptual variety requires widely integrating approach.

This paper attempts to meet this challenge by bringing together three ideas. Namely, it takes mathematical structure of qubit used for cognitive-behavioral modeling Busemeyer and Bruza (2012); Surov and Alodjants (2018), employs it for process-semantic representation of contexts as described in Surov (2021b), and establishes correspondence of the resulting model with fundamentals of emotion science. Conceptually, the obtained model aligns with the core affect and psychological construction framework Barrett and Russell (2015), while its geometrical expression resembles valence-arousal circumplex Russell (2009). There are, however, principal differences resulting in elaboration of the classical notion of core affect, as reflected by the title of this work.

The paper is organized as follows. Section 2 highlights features of emotion that are central for the following consideration. Sections 3 and 4 describe the theory and 
report experimental verification. Section 5 discusses relation of the theory with basic approaches to emotion modeling. Section 6 provides methodological and practical outlook.

\section{Features of emotion in focus}

Prognostic function, subjectivity, contextuality, and goal-orientedness are acknowledged features of emotions. Although well-known individually and qualitatively, their modeling in a single mathematical structure was not achieved before. These features are summarized in this section preparing for the following theory.

\subsection{Prognostic function and process representation}

Productive view of emotions considers them as essential aspect of cognition, facilitating its central activity of behavioral control according to the needs of an organism and species Plutchik (1980). This is achieved by simulation of internal and external processes in prediction-correction scheme including prognosis of the future, setting goals, and comparing outcome of the performed action with the anticipated result Barrett and Simmons (2015); Carver and Scheier (1990); Dayan (2012); Grush (1997). Starting from cellular level, most of this activity goes in automated unconscious mode; only small part of high-level representations are available to conscious as subjective states categorized to fear, anger, joy, and other classes Kellerman (2020). These emotional terms label physiological algorithms tuning the whole system in a coherent mode appropriate for anticipated events Kelly (2003); Oatley and Johnson-Laird (1987).

Emotions are considered as a basic system of pro-active behavioral control, preceding and underlying refined forms of conceptual "non-emotional" thinking Hoey, Schröder, and Alhothali (2016). As long as perception confirms the expected scenario, cognition is reduced to the economical monitoring mode. Energy-consuming affective states are activated as correction signals when ongoing plan is disrupted and transition of an organism to a different kind of action is necessary Johnson-Laird and Oatley (1992).

Ability to predict future events requires cognitive reflection of causal links underlying both environmental and internal organismic processes of a subject. Necessary robustness of natural behavior-controlling systems requires cognitive models responsible for that to be versatile and simple; this is achieved by predictive coding of information central for natural cognition Bubic, Yves von Cramon, and Schubotz (2010); Clark (2013); Gładziejewski (2016); Greve (2015). Emotional categories are considered as crucial part of this causal-predictive coding and signaling system Barrett (2017); Gendron, Mesquita, and Barrett (2020). Fear, for example, categorizes perception as uncontrolled process possibly leading to disaster, with plausible coping strategies being escape or hiding Mobbs et al. (2019).

\subsection{Contextuality and goal-orientedness}

A snake is scary for someone who has stepped on its tail, and far less so at fair distance. Alternatively, less fear would come up in someone ensured that it is not poisonous, or by a child knowing nothing about snakes. Thus, although emotion is 
often perceived as focused on a particular object, it accounts for a whole situation around a subject - the context often resisting (object-wise) decomposition Conway and Bekerian (1987); Keltner, Sauter, Tracy, and Cowen (2019); Mesquita, Barrett, and Smith (2010); Zajonc (1980). As in the above example, the context includes not only spatial environment, but also internal variables of a subject like knowledge or culture.

Refined version of contextuality distinguishes subjective goal and planning structure as a crucial type of contextual information. Reason for this is that valuation of sensory input during construction of emotions is inherently goal-directed Anderson (1989); Fink and Yolles (2018); Gross (2015); Scherer (1982); Solomon (2003). Within prognostic modeling activity, major emotion classes are associated with distinct goals and juncture types in multi-goal planning sequences, facilitating transition to a new phase of planned activity Johnson-Laird and Oatley (1992); Oatley and Johnson-Laird (1987). Continuing the above example, finding a snake could finalize effort of a zoologist to his joy or satisfaction instead of fear. This conditioning of emotions by subjective goals, considered as primary determinant of emotional phenomena Moors and Fischer (2019) is central to the model described below.

\subsection{Pragmatic and subjectively-semantic nature}

Although genesis and function of emotions is largely social Boiger and Mesquita (2012); Doyle McCarthy (1994); Mesquita (2010); Plutchik (1983); Scarantino (2017b), their construction and maintenance is personal duty Lazarus (1995). Emotions are conceptualized as individual's engagement with the world, in which sensory input is evaluated according to subjective norms relative to subjective goals and plans Lazarus (1991); Novacek and Lazarus (1990); Solomon (2003).

By representing sensory information according to interests, values, goals, and plans of a subject, emotions have direct behavioral meaning and use. This is pragmaticsemantic quality of emotions, absent in objective sensory data Kolchinsky and Wolpert (2018); Kuznetsov, Baksanskii, and Zholkov (2012). Accordingly, generation of emotions is a meaning-construction process in which subject actually makes sense of its behavioral environment Peil (2014). This pragmatic, purposeful, and thereby "semantic" quality is fundamental for natural cognitive systems Ackoff and Emery (2017); Barrett (2017); De Jesus (2018); Tarski (1944).

Subject-centered, pragmatic cognition is also required by severe energetic restrictions imposed on living organisms. Only vital parts of behavior-controlling system could be kept onboard, with all excessive, unstable, practice-irrelevant, and otherwise inefficient cognitive functions extinguished by competitive historical process together with their carriers. Accordingly, view of emotions as trouble-making facility of mind compromising correct logical thinking is out of question Cohen (2005); Plutchik (1980). Adequate model would include emotions as integral feature of human cognition, expanding outdated concepts of rationality Corr (2013); ?.

\section{Quantum theory of affect semantics}

The theory is developed in three steps. First step describes necessary mathematical structure, based on the qubit model of context representation developed in Surov (2021a). Here, it is generalized to the case of mixed states and supplemented by the concept of observables. Next Section 3.2 introduced process-semantic interpretation of 
the qubit state space developed in Surov (2021b). Based on these ingredients, theory of affect semantics is described in Section 3.3.

\subsection{Basic terms}

Mathematics described in this section is standard quantum-theoretic routine. Example textbooks presenting varying approaches to it are Jaeger (2007); Le Bellac (2006); Nielsen and Chuang (2010).

\subsubsection{Pure states}

Consider a subject bound to make a choice between two mutually exclusive cognitivebehavioral alternatives: DO or NOT DO, TRUE or FALSE, YES or NO, etc. All information used to make this decision, called context, is represented by the subject by pure qubit state

$$
|\psi\rangle=c_{0}|0\rangle+e^{i \phi} c_{1}|1\rangle=\cos \frac{\theta}{2}|0\rangle+e^{i \phi} \sin \frac{\theta}{2}|1\rangle,
$$

where 0 and 1 label the alternatives, $c_{i}$ are real-valued coefficients, and $\phi$ is phase parameter. Angle brackets $|\cdot\rangle$ denote column vectors, so that qubit state $|\psi\rangle$ is a vector in two-dimensional space formed by basis vectors $|0\rangle$ and $|1\rangle$ :

$$
|0\rangle=\left[\begin{array}{l}
1 \\
0
\end{array}\right], \quad|1\rangle=\left[\begin{array}{l}
0 \\
1
\end{array}\right], \quad|\psi\rangle=\left[\begin{array}{c}
c_{0} \\
e^{i \phi} c_{1}
\end{array}\right] .
$$

With coefficients $c_{i}$ parametrized via (polar) angle $\theta$, state (1) is visualized by a unit vector pointing from the origin of a three-dimensional cartesian space to the surface of a unit-radius (Bloch) sphere as shown in Figure 1.

Coefficients $c_{i}$ encode subjective favorability of the represented context for choosing corresponding alternatives. This favorability is quantified by decision probabilities

$$
p_{i}=|\langle\psi \mid i\rangle|^{2}=c_{i}^{2}, \quad p_{0}+p_{1}=\cos ^{2} \frac{\theta}{2}+\sin ^{2} \frac{\theta}{2}=1,
$$

defined statistically for an ensemble of identically staged experiments. In the above expression $\langle\psi \mid i\rangle$ denotes inner product of basis vector $|i\rangle, i=0,1$ with complex-conjugate (Hermitian) transpose of $|\psi\rangle$ :

$$
\langle\psi|=| \psi\rangle^{\dagger}=\left[\begin{array}{ll}
c_{0} & e^{-i \phi} c_{1}
\end{array}\right] .
$$

Unlike standard Euclidean geometry, orthogonality of the qubit states corresponds to opposite orientation of their vectors, as seen e.g. for basis states $\langle 0 \mid 1\rangle=0$ located at the poles of the sphere.

Unity sum of the probabilities in (3) expresses the restriction that exactly one of the alternatives is chosen in each experiment. In contrast to $c_{i}$ and $\theta$, phase $\phi$ is not uniquely determined by observable decision statistics. This quantity, represented by azimuthal dimension of the Bloch sphere, allows subject to build multiple context representations describing the same decision probabilities. 


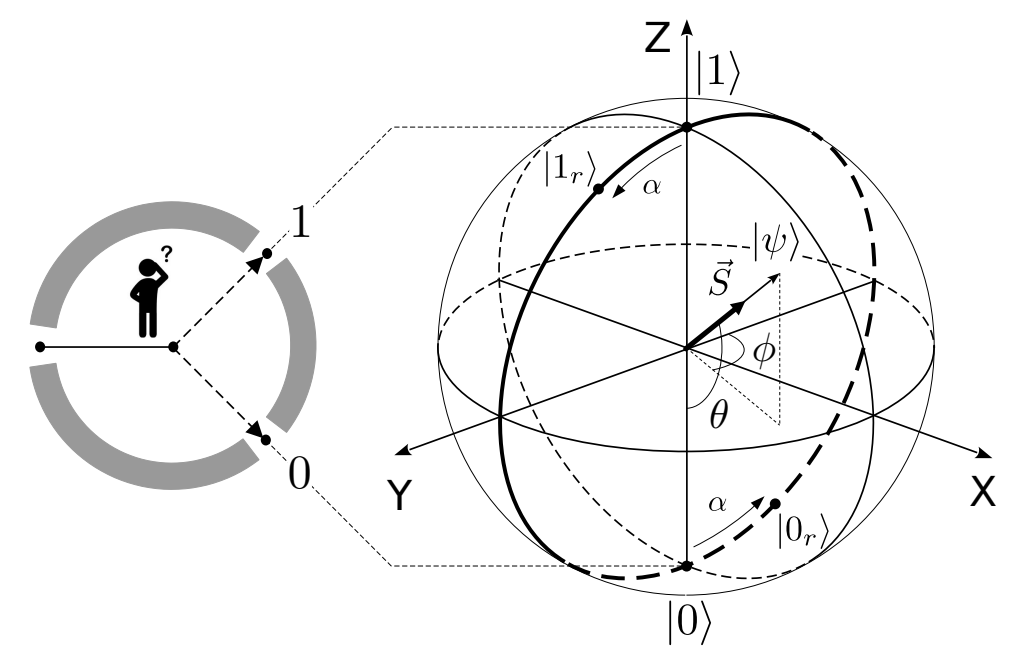

Figure 1. Quantum model of context representation relative to the binary decision alternative faced by a subject. The context is represented by the qubit state vector within the unit-radius Bloch sphere built on the poles identified with the basis alternatives 0,1 . Pure representation (1) corresponds to the unit-length vector defined by polar and azimuthal angles $\theta$ and $\phi$, while mixed representation corresponds to shorter vector (7) pointing to the interior of the sphere. States $\left|1_{r}\right\rangle,\left|0_{r}\right\rangle$ (9) represent rotated observable $\hat{O}(\alpha, \beta=0)(10)$.

\subsubsection{Mixed states}

Theory described above accounts for an ideal situation when basis behavioral alternatives are well defined, subject is in full control over his choice and is able to include the perceived context in a single cognitive representation Surov (2021b). In realistic cases, however, several representations (1) are blended with probability-weights $P_{i}$ in a mixture state

$$
\hat{\rho}=\sum_{i} P_{i}\left|\psi_{i}\right\rangle\left\langle\psi_{i}\right|, \quad \sum_{i} P_{i}=1
$$

As follows from function of outer products $\left|\psi_{i}\right\rangle\left\langle\psi_{i}\right|$, this object is two-by-two matrix. It is always representable in the form

$$
\hat{\rho}=\frac{1}{2}\left[\begin{array}{cc}
1+s_{z} & s_{y}-i s_{x} \\
s_{y}+i s_{x} & 1-s_{z}
\end{array}\right]
$$

with $s_{\mu}$ being components of a three-dimensional real-valued (Stokes) vector

$$
\vec{S}=\left[\begin{array}{c}
s_{x} \\
s_{y} \\
s_{z}
\end{array}\right], \quad|\vec{S}|^{2}=s_{x}^{2}+s_{y}^{2}+s_{z}^{2} \leq 1
$$

Mixed state (5)-(7) generalizes pure state (1). Geometrically, this corresponds to using Stokes vectors (7) occupying interior of the Bloch sphere along with its surface. The latter exclusively corresponds to pure states for which inequality (7) is saturated.

Diagonal of matrix (6) contains decision probabilities $\left(1 \pm s_{z}\right) / 2$ generalizing pure state case (3). As seen from this expression, decision probabilities are proportional to lengths of the sphere's diameter segments produced by projection of the state vector (1) or (7). In the mixed case, components $s_{x, y}$ determine direction of state vector in 
the azimuthal (equatorial, XY) plane given by the phase parameter $\phi$ as in pure case.

Useful metric in the qubit state space is trace distance defined for any two states $\hat{\rho}_{1,2}$ as

$$
\begin{aligned}
& D\left(\hat{\rho}_{1}, \hat{\rho}_{2}\right)=\frac{\operatorname{Tr}\left|\hat{\rho}_{1}-\hat{\rho}_{2}\right|}{2}=\frac{\left|\vec{S}_{1}-\vec{S}_{2}\right|}{2} \\
& |\hat{\rho}|=\sqrt{\hat{\rho}^{\dagger} \hat{\rho}}, \quad 0 \leq D \leq 1
\end{aligned}
$$

where trace $\operatorname{Tr}$ returns sum of diagonal elements of the input matrix. As indicated by the latter expression, (8) is equal to half of the ordinary Euclidean distance between ends of the corresponding Stokes vectors (7) in Bloch representation Nielsen and Chuang (2010).

\subsubsection{Observables}

Representation of contexts in the Bloch ball allows to prognose probabilities for other decision alternatives, accommodated in the same Hilbert space. This is achieved by modifying the measurement basis, while context representations are kept in place. In Bloch representation this corresponds to rotation of the sphere's diameter 1-0, with initial basis alternatives $|1\rangle,|0\rangle$ moving on the surface. New positions of these states are represented by orthogonal vectors

$$
\begin{aligned}
& \left|0_{r}\right\rangle=\cos \frac{\alpha}{2}|0\rangle+e^{i \beta} \sin \frac{\alpha}{2}|1\rangle \\
& \left|1_{r}\right\rangle=\sin \frac{\alpha}{2}|0\rangle-e^{i \beta} \cos \frac{\alpha}{2}|1\rangle,
\end{aligned}
$$

defined analogous to the pure state (1). With $\beta=0$, for example, diameter rotates by angle $\alpha$ in the YZ section of the Bloch sphere, producing states (9) shown in Figure 1. Probabilities of choosing new alternatives in the same context (1) are found by rule (3) with $|i\rangle$ replaced by $\left|i_{r}\right\rangle$ (9).

Decision alternatives (9) are usually associated with real numbers $v_{0,1}$ quantifying e.g. their subjective value. Decision-making experiments with ensemble of subjects prepared in identical contexts then are equivalent to sampling of binary random variable. If the context representation is pure (1), mean of this variable is equal to values $v_{i}$ weighted by probabilities (3). In the mixed context (6), the same mean value is computed as

$$
m(\alpha, \beta)=\operatorname{Tr}[\hat{\rho} \cdot \hat{O}(\alpha, \beta)], \quad \hat{O}=v_{0}\left|0_{r}\right\rangle\left\langle 0_{r}\left|+v_{1}\right| 1_{r}\right\rangle\left\langle 1_{r}\right| .
$$

where matrix $\hat{O}(\alpha, \beta)$ represents the observable defined by outcomes $\left|i_{r}\right\rangle$ and values $v_{i}$, and $\cdot$ denotes matrix multiplication. With $\alpha=\pi / 2, \beta=0$ producing basis vectors (9) directed along $\mathrm{Y}$ axis, and $v_{i}= \pm 1$, for example, formula (10) produces $m=s_{y}$ being Y-component of vector (7). 


\subsection{Semantics of the qubit space}

\subsubsection{Process phase dimension}

Complex exponent $e^{i \phi}$ is periodic function repeating itself after every $2 \pi$ radians or, equivalently, 360 degrees. This defines circular topology of the phase dimension suitable for mapping of process-causal relations between contexts Surov (2021b). In this scheme, contexts are mapped to the azimuthal phase dimension $0^{\circ} \leq \phi \leq 360^{\circ}$ according to their subjective affiliation with particular functional classes relative to the basis decision alternative. Six basic classes are defined as follows:

1 Sensing, $330^{\circ}<\phi<30^{\circ}$

Contexts describing circumstances and observations leading to the basis decision alternative. For a choice whether to build a new house (1) or not (0) this could be, for example, aging of or negative feeling about the existing construction, anticipation of new residents or environmental pressures, etc.

2 Novelty, $30^{\circ}<\phi<90^{\circ}$

Contexts describing a particular novel factor (surprise, issue, problem) addressed by the considered decision. Continuing the above example, this could be destruction of the former house, growing of the family, etc.

3 Goal-plan, $90^{\circ}<\phi<150^{\circ}$

Contexts setting objectives regarding the novelty and describing plans for its achievement. With the goal of increasing or improving the living space, the plan could include funding strategy, architecture, floor plan, schedule of the works, etc.

4 Action, $150^{\circ}<\phi<210^{\circ}$

Contexts describing efforts for implimentation of the plan, including preparation of the resources and building process with all associated activities.

5 Progress, $210^{\circ}<\phi<270^{\circ}$

Contexts describing intermediate advances providing feedback for the action. This includes finishing paperwork and legal documents, underground connections, building frame, interior works, plumbing etc. with corresponding inspections and certifications.

6 Result, $270^{\circ}<\phi<330^{\circ}$

Contexts describing final results and consequences of the decision. Images of the new house, novel possibilities for living activity, change of social relations and environment, other results and implications are mapped to this phase range.

Among these classes, Novelty, Action, and Result constitute an archetypal triple of process stages recognized in the classical narrative and screenplay structures, cybernetic control loops, and variety of life-cycle models Surov (2021b). This triple forms minimal closed semantic structure such that Novelty follows from the previous Results and requires Action, Action is a move from Novelty to the Result, while Result is a consequence of previous Action and potential cause of future Novelty.

Goal-plan, Progress, and Sensing account for less expressive, but distinct parts of behavioral cycle enabling transitions between three main stages. The resulting sixstage process taxonomy is considered optimal for behavioral control due to matching with normal capacity of human attention able to capture no more than $7 \pm 2$ items simultaneously Miller (1956).

Process-stage categorization of the qubit's azimuthal dimension is shown in Figure 2(a). This process structure holds both for pure and mixed qubit states, mapped 

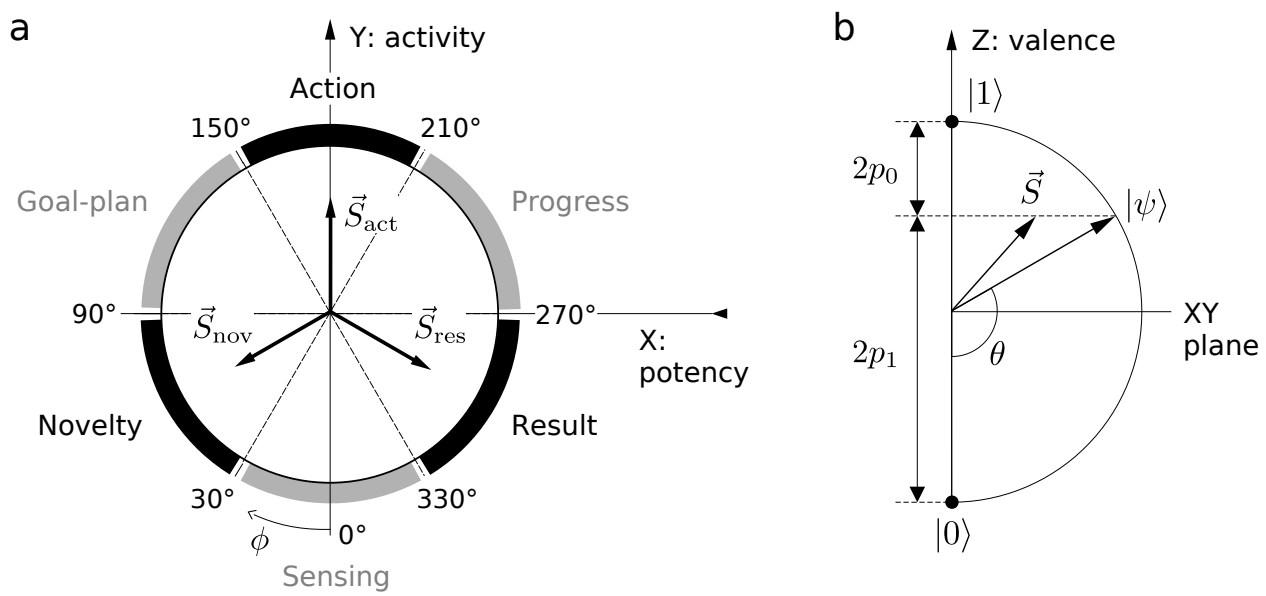

Figure 2. a: Process-semantic context classes Sensing, Novelty, Goal-Plan, Action, Progress, and Result, mapped to the azimuthal dimension (XY plane) of the Bloch sphere shown in Figure 1. Pure (1) and mixed states (7) of each class occupy perimeter and interior of the corresponding sector. $\mathrm{X}$ and $\mathrm{Y}$ are potency and activity axes with maximal values at $\phi=90^{\circ}$ and $\phi=180^{\circ}$ respectively. b: Vertical section of the Bloch sphere showing $\mathrm{Z}$ axis encoding subjective valence of context in qubit representation. Positive and negative evaluation corresponds to $\theta \gtrless 90^{\circ}$ for pure states and $s_{z} \gtrless 0$ for mixed states. In both cases, probabilities of choosing basis alternatives are proportional to segments of the diameter divided by projection of state vector.

to the circumference and interior of the azimuthal circle, respectively. Circular process dimension associated with the basis behavioral alternative $0 / 1$ is a key difference from classical probability space describing binary uncertainty. The latter corresponds to the diameter of the Bloch sphere shown in Figure 2(b).

Arrangement of qubit context representations in the azimuthal phase dimension expresses causal structure, ascribed by the subject to behavioral contexts. In the above example, one could consider building the same house for the joy of the process itself, or to compete with a neighbor; in each hypothetical causal structure, set of the relevant contexts would be different. Abstract process cycle thus functions as an empty semantic template, populated by particular contexts in each decision case according to their causal relations established by the subject.

\subsubsection{Meaning of Cartesian axes}

Process-stage structure of the azimuthal dimension entails interpretation of $\mathrm{X}$ and $\mathrm{Y}$ axes of the equatorial plane of the Bloch sphere as Potency and Activity as shown in Figure 2(a).

Namely, the most behaviorally-influencing contexts are those describing goals of a subject regarding the basis decision. Such contexts, positioned near $\phi=90^{\circ}$ of the process cycle, oppose Progress and Result-class contexts at which ability of a subject to influence the process is minimal. This contrast, alternatively formulated as freedom versus stability, defines horizontal $\mathrm{X}$ axis called Potency.

$\mathrm{Y}$ axis discriminates contexts according to their Activity. Upper half of the process cycle accommodates (externally) active contexts, while contexts in the lower half are (externally) passive. In the process cycle, activity rises above zero from setting goals and developing plans near $\phi=90^{\circ}$, reaches maximum at zenith $\phi=180^{\circ}$, declines upon getting the result near $\phi=270^{\circ}$ and drops to minimum in sensing stage centered at $\phi=0^{\circ}$.

As noted in Section 3.1, contexts are also ranked according by their favorability for 
the basis behavioral alternative. This subjective estimation, quantified by probabilities (3) in the pure case and diagonal elements of density matrix in the mixed case, defines vertical position of the qubit state in the Bloch sphere as shown in Figure 2(b). Vertical $\mathrm{Z}$ axis thus discriminates contexts according to their subjective evaluation in each process-stage class. For example, objective needs could be considered as proper reason for building a house, in contrast to luxury competition possibly considered in the same quality as vicious; in the action class, opposite valuations could be ascribed to achieving the goal by robbery and by subject's own labor.

\subsection{Quantum model of emotions}

Sections 3.1 and 3.2 indicate that qubit representation of contexts naturally embodies features of emotions summarized in Section 2. In particular,

- Contextuality follows from definition, as qubit states represent whole contexts instead of separate objects or features;

- Goal-orientedness is ensured by construction of representation space based on particular behavioral choice to be made;

- Prognostic function is provided by qubit's azimuthal process dimension reflecting subjective causal links and planning structure;

- Subjectivity is also ensured by construction. Necessary degree of freedom is provided by azimuthal dimension of the qubit, allowing the same decision probabilities to be modeled by multiple representations of contexts.

This affinity is the basis for modeling emotions by qubit states.

\subsubsection{Process-value classes of emotion}

Quantum model of emotions follows from semantics of the qubit space described in Section 3.2. Its crucial elements are process-stage map of the qubit's azimuthal dimension and $\mathrm{Z}$ axis of the Bloch sphere encoding subjective evaluation of contexts. Namely, context of each process stages are subjectively reflected to a particular class of qubit-emotion states, further discriminated to positive or negative valence:

(1) Sensing: Anticipation - Anxiety

In positive way, reflecting and observational activity of Sensing stage is facilitated by calm and serene emotions, possibly accompanied with positively connoted anticipation of the future. In negative way, the same activity is accompanied by melancholy and depression, while anticipation takes form of anxiety.

(2) Novelty: Surprise - Fear

By definition, unexpected information constituting Novelty drops out of the expected, normal course of events, producing error signal within predictioncorrection control loop (Section 2.1).

By virtue of its disturbing and often threatening implications, default evaluation of Novelty is negative, with corresponding subjective experiences called worry, fear, terror, dread, etc. Positively evaluated novelty produces wonder, amazing, astonishing, surprise-kind states.

(3) Goal-plan: Inspiration - Boredom 
Due to its deliberate and intellectual nature, this stage is weakly emotional. In positive way, setting goals and drawing plans is accompanied by inspiration, vigilance, passion, and excitement. In negative way, the same contexts are experienced as boredom or malice.

(4) Action: Zeal - Rage

Extensive activity of this stage requires mobilization of the organism's resources and skills. If evaluated positively, this activity is accompanied by ambition, passion, courage, zeal, etc. In the negative case, the action goes in destructive way with emotions of anger, hatred, and contempt.

(5) Progress: Acceptance - Disgust

Depending on the evaluation of intermediate results and feedback received at this stage, contexts of this class are reflected by emotions of acceptance, elation, disappointment, or disgust.

(6) Result: Joy - Sadness

Subjective reflection of contexts in this class provides assessment of the result, crucial for behavioral control. Positively feedback is provided in the form of contentment, joy, happiness, bliss and similar states, while negatively evaluated result is experienced as sadness, grief, dismay, and the like.

As seen from this list, process stages are not equal in emotional expressiveness. Novelty, Action, and Result are emotionally strong, while Sensing, Goal-Plan, and Progress are relatively weak, in agreement with the division of process stages to main and intermediate triples. Alteration of emotional expressivity is related to variation of the activity during the process cycle. Namely, subject's cognitive and physiological functions in strongly emotional stages differ from their precursors dramatically:

- processing of Novelty requires mobilization and cognitive focus absent in Sensing stage;

- effective Action requires shift from visionary thinking at the Goal-plan stage toward down-to-earth implementation;

- adequate assessing of the Result requires balanced feeling hardly possible in making the Progress.

Onset of weakly emotional stages, in contrast, does not require significant psychophysiological reconfiguration:

- setting Goals and Plans is cognitive activity compatible with detection and recognition tasks of Novelty stage;

- Progress continues energetic implementation activity initiated at Action stage;

- Sensing is close to defocused mode of feeling used to estimate the Result at the previous stage.

Most pronounced emotion states, synchronized with major juncture points of planning sequences (Section 2.2), are therefore expected at the onsets of three main process stages as indicated by vectors in Figure 2(a).

\subsubsection{Map of qubit-emotional states}

The above classes of emotions are located in the Bloch sphere according to schemes shown Figure 2(a). Namely, equatorial plane is divided to six azimuthal sectors of 


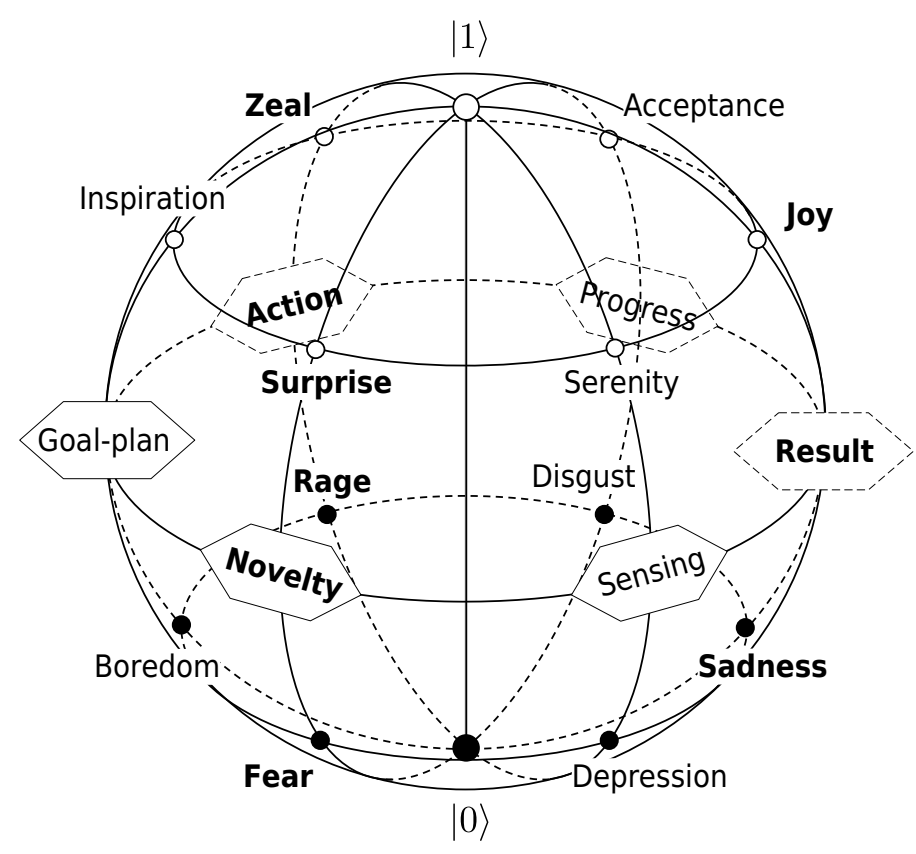

Figure 3. Map of qubit-emotional states relative to the basis dichotomic alternative $0 / 1$ in the Bloch sphere representation. Emotion classes are defined by six process stages categorizing equatorial circle according to Figure 2. Positive and negative-valued emotion prototypes marked by filled and empty dots are discriminated by vertical (polar) dimension. Strongly emotional stages are indicated in bold.

$60^{\circ}$ each corresponding to Sensing, Novelty, Goal-plan, Action, Progress, and Result classes, with upper and lower hemispheres containing positive and negative emotions.

The resulting spherical structure is sketched in Figure 3, with emotion classes represented by prototype points centered at the corresponding process stages. In each class, positive and negative-valued items are discriminated in polar $(\theta)$ dimension according to Figure 2(b). For example, difference in evaluation between Anxiety and Anticipation is quantified by polar angles $\theta_{\text {anxiety }}<90^{\circ}$ and $\theta_{\text {anticipation }}>90^{\circ}$. Both emotions, accompanying transition from Sensing to Novelty, have the same azimuthal phase $\phi=30^{\circ}$.

12 emotional prototypes include 6 negative and 6 positive ones, marked in Figure 3 by filled and empty dots. In positive way, Sensing leads to Novelty via surprise, Novelty leads to Goal-plan via inspiration, Goal-plan proceeds to Action with zeal, Action proceeds to Progress via acceptance, and Progress turns to Result with joy, and Result is followed by new Sensing with serenity. In case of negative evaluation, the same process stages are accompanied by fear instead of surprise, boredom instead of passion, rage instead of zeal, disgust instead of acceptance, sadness instead of joy, and depression instead of serenity ${ }^{1}$.

In Figure 3, positions of emotion prototypes on the sphere's surface are chosen arbitrary for readability of the scheme. In practice, contexts are represented by mixed qubit-emotional states (Section 3.1.2) occupying interior of the Bloch sphere, divided to the same azimuthal sectors. According to similarity measure (8), distinction between

\footnotetext{
${ }^{1}$ Connectivity of the process cycle requires that no stage can be skipped or jumped over. Accordingly, resolution of any binary uncertainty involves successive passing through all process stages, with azimuthal phase $\phi$ progressing from $0^{\circ}$ to $360^{\circ}$. The only available options are (i) evaluation just discussed, functioning as positive or negative feedback signal of varying intensity Carver and Scheier (1990), and (ii) whether these signals will be recognized as emotional experiences.
} 
different emotions decreases with moving from the surface to the center of the sphere. For fixed evaluation and azimuthal phase, this change is quantified by length of the corresponding vector $\vec{S}$ in the XY plane as illustrated in Figure 2(b). This length $\left(s_{x}^{2}+\right.$ $\left.s_{y}^{2}\right)^{1 / 2}$, constrained by (7), encodes intensity of emotional states. It goes to zero at the diameter of the Bloch sphere, visualizing classical probability space and representing non-emotional, objective part of cognitive information Surov (2021b).

\section{Experiment}

The theory described above is supported by two experimental approaches. First, the expected structure of emotional terms is found in the word2vec model of English language $^{2}$. Idea of the experiment, calculation, and the obtained results are described in Sections 4.1-4.3. Second, the same structure is observed in emotional spaces constructed in previous experimental studies, Section 4.4.

\subsection{Idea of the experiment}

As shown in Surov (2021b), word2vec data contain process-semantic structure described in Section 3.2. Namely, high-dimensional word2vec space contains a plane (that is, two-dimensional subspace) in which process-related terms specific for six the process stages, e.g.

(1) reflection, attention, observation, ...

(2) puzzle, question, surprise, ...

(3) purpose, concept, plan, ...

(4) implement, work, strive, ...

(5) achieve, gain, reach, ...

(6) completion, record, aftermath, ...

form distinct circularly ordered clusters as pictured in Figure 2(a). If, according to the above theory, emotional states are derived from this process structure, then corresponding terms have to follow the expected ordering in the same process-semantic plane (corresponding to equatorial circle of emotional map shown in Figure 3). Two following subsections test this prediction.

\subsection{Construction of qubit semantic space from word2vec data}

\subsubsection{Finding the process-semantic plane}

The process-semantic plane is found in word2vec representation space by method described in Surov (2021b) with database of 300-dimensional real-valued vectors taken from Google Code Archive (2013). Three main process-stage classes Novelty, Action, and Result are populated by $N=12$ words each:

- Novelty, $k=$ nov:

Novelty, concept, distinguish, factor, question, unknown, puzzle, note detect, doubt, reason, idea;

\footnotetext{
${ }^{2}$ Word2vec is a model of natural language, capturing regularities of word usage in high-dimensional vectors ascribed to each word Mikolov, Chen, Corrado, and Dean (2013).
} 
- Action, $k=$ act:

Action, work, fight, battle, force, strive, compete, cooperate, execute, engage, energy, effort;

- Result, $k=$ res:

Result, end, victory, defeat, value, aftermath, estimate, record, harvest, outcome, conclusion, completion.

For each process class $k$, mean of individual word vectors $\vec{v}_{k, i}$ defines real-valued prototype vector

$$
\vec{V}_{k}=\frac{1}{N} \sum_{i=1}^{N} \vec{v}_{k, i}
$$

Basis vectors $\vec{X}$ and $\vec{Y}$ defining the process plane are then found as real and imaginary components of a single 300-dimensional complex-valued vector

$$
\vec{X}=\operatorname{Re}[\vec{\Omega}], \quad \vec{Y}=\operatorname{Im}[\vec{\Omega}], \quad \vec{\Omega}=\frac{1}{n_{a}} \sum_{k} \vec{V}_{k} e^{i \phi_{k}},
$$

where $n_{a}$ is normalization constant and azimuthal positions of stage prototypes

$$
\phi_{\mathrm{nov}}=60^{\circ}, \quad \phi_{\mathrm{act}}=180^{\circ}, \quad \phi_{\mathrm{res}}=300^{\circ}
$$

are defined according to Figure 2(a).

\subsubsection{Projection to process-semantic plane}

Any word2vec representation $\vec{w}$ is projected to the process-semantic plane by taking scalar product with vector $\vec{\Omega}(12)$. Real $s_{x}$ and imaginary $s_{y}$ parts of the resulting complex number

$$
s_{x y}=\vec{\Omega} \cdot \vec{w}=s_{x}+i s_{y}
$$

are coordinates of this projection in the process-semantic plane. They are identified with the first two Stokes parameters, so that complex number (14) defines projection of the mixed qubit state vector (7) to the azimuthal (XY) plane of the Bloch sphere.

Projection (14) allows e.g. to assess the process-plane finding procedure described in Section 4.2.1. With $\vec{V}_{k}$ and $\vec{v}_{k, i}(11)$ taken as $\vec{w}$ in (14), one obtains positions of central prototypes and individual words for each process-semantic class $k$ in the process plane:

$$
S_{k}=\vec{\Omega} \cdot \vec{V}_{k}, \quad s_{k, i}=\vec{\Omega} \cdot \vec{v}_{k, i} .
$$

With Novelty, Action, and Result labeled by blue, red, and green, these positions are shown in Figure 4. Obtained vectors $S_{k}$ deviate from the intended azimuthal angles (13) by $4^{\circ}$ on average, with individual words deviating from their prototypes by $12^{\circ}$ on average, ensuring proper azimuthal resolution. This indicates success of the procedure (11)-(13).

Prototype vectors (15) agree with theoretical layout shown in Figure 2(a) not only in directions, but also in length: among the three, modulus $\left|S_{k}\right|$ varies by $1.5 \%$ on average. Mean of these distances is normalized to unity by constant $n_{a}$ in (12). 


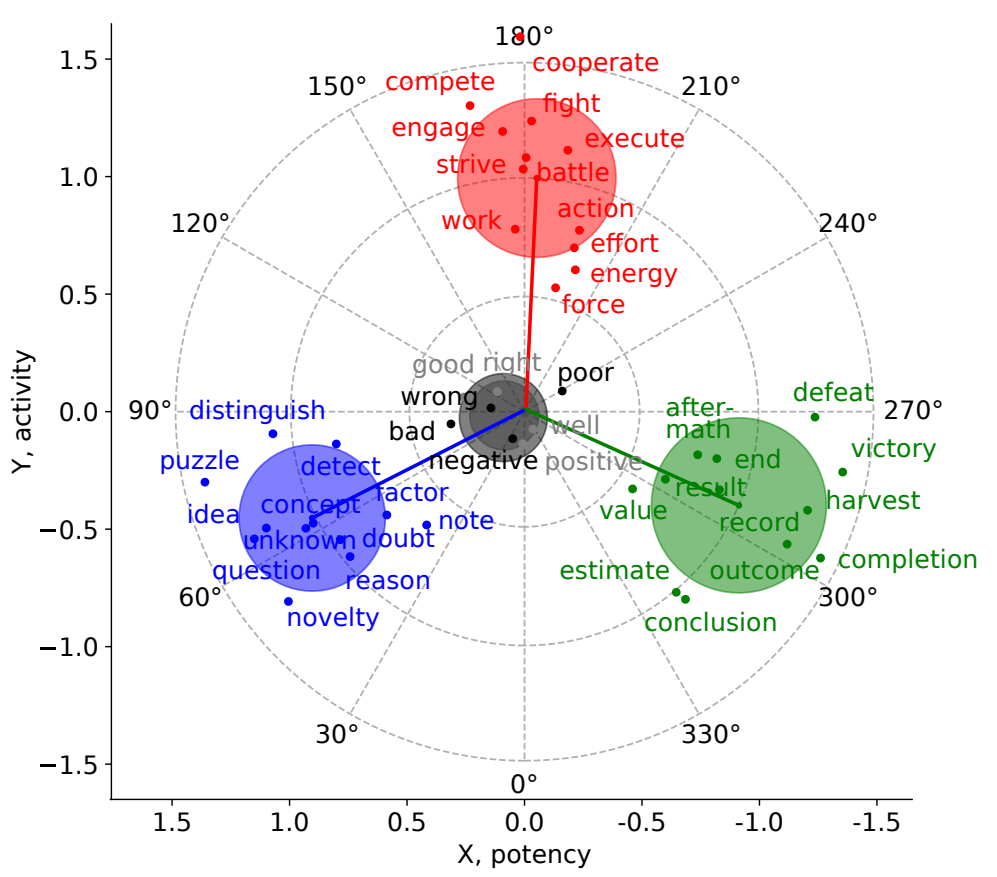

Figure 4. Five prototypes locating the (context-averaged) qubit semantic space in 300-dimensional word2vec space, in projection to the process-semantic plane $\vec{\Omega}(15)$. Process-semantic prototypes Novelty, Action, and Result defining plane $\vec{\Omega}(12)$ are composed of individual words shown in blue, red, and green, respectively. Positive and Negative prototypes defning evaluation $\mathrm{Z}$ axis (16) are shown in gray and black. Lines and circles denote prototype centers and scattering of the corresponding individual words.

\subsubsection{Evaluation $Z$ axis}

Evaluation axis is defined in word2vec space as real-valued 300-dimensional vector

$$
\vec{Z}=\frac{1}{n_{z}}\left(\vec{V}_{1}-\vec{V}_{0}\right)
$$

combining positive and negative prototypes $\vec{V}_{1}$ and $\vec{V}_{0}$, normalized by constant $n_{z}$. Prototypes $\vec{V}_{0,1}$ are constructed according to (11) from the following words:

- Positive (1):

Positive, good, well, right;

- Negative (0):

Negative, bad, poor, wrong.

As expected, these prototypes corresponding to the poles of the Bloch sphere are nearly orthogonal to the process plane $\vec{\Omega}$, locating close to the center of Figure 4 .

Evaluation of any word2vec representation $\vec{w}$ is given by its projection to $\mathrm{Z}$ axis, found as scalar product

$$
s_{z}=\vec{Z} \cdot \vec{w}
$$

analogous to the process plane (14). For example, evaluations of the process-stage prototypes $\vec{V}_{\text {nov }}, \vec{V}_{\text {act }}$, and $\vec{V}_{\text {res }}$ equal to $-0.12,0.17$, and -0.08 respectively. These prototypes thus are close to the equatorial plane of the Bloch sphere as in scheme on Figure 3. Z projection (17) complements XY component (14) to full three-dimensional 
Stokes vector (7) defining qubit-emotional state.

\subsection{Qubit space of emotion terms}

\subsubsection{Process-semantic map}

Figure 5 shows 98 emotion-relevant terms projected to the process-semantic plane as described in Sections 4.2.2 and 4.2.3. According to the theory, Novelty-, Action-, and Result-class emotions are expected in blue, red, and green sectors of the plane, centered at angles (13) and spanning range of $120^{\circ}$ each.

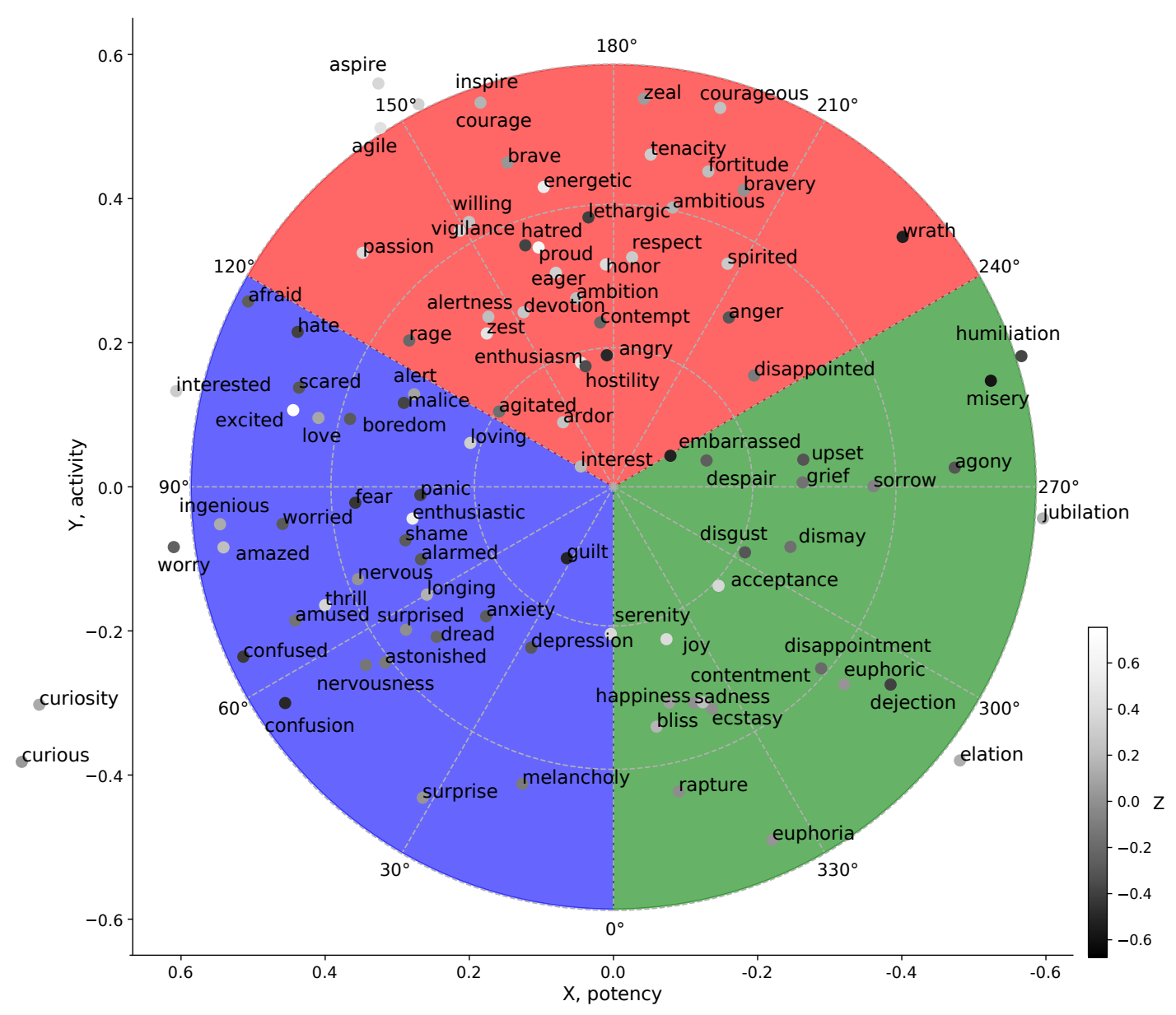

Figure 5. Process-semantic map of 98 emotion-related terms extracted from word2vec data. Position of each term is obtained as projection (14) of the corresponding word2vec vector to the process-semantic plane $\vec{\Omega}$ constructed by non-emotional prototypes shown in Figure 4. Evaluation of each term computed by projection of the same vector to $\mathrm{Z}$ axis (17) is shown by grayscale color. Blue, red, and green sectors denote Novelty, Action, and Result azimuthal ranges according to Figures 2 and 3.

This expectation is generally fulfilled.

Fear, anxiety, and surprise-like terms are correctly mapped to the blue sector. Stability of the individual word's positions is evidenced by considering various synonymous and grammatical forms. For example, surprise - surprised, confuse-confusion, interested - curious, anxiety - worry - worried alarmed - nervous - nervousness all consistently fall to this sector. Pairs divided 
between blue and red sectors, e.g. alert - alertness, excited - agitated, hate - hatred, love - passion, enthusiasm - enthusiastic, are mainly located close to the border.

Except for borderline cases of this kind, emotions of red and green sectors terms also align with expectation. Red sector includes angry - anger - wrath

- inflamed - rage - spite, aspire - inspire - spirited, passion devotion - zest - zeal - ardor - eager, brave - bravery - fearless - courage - courageous - fortitude - tenacity, ambition - ambitious - honor - proud - respect and alertness - agile - vigilance groups. Green sector consistently accommodates sorrow - misery - grief - despair - dismay - agony, acceptance - disgust, joy - serenity - contentment melancholy - sadness - disappointment - dejection - upset, happiness - bliss - rapture - ecstasy - euphoria - elation - jubilation groups. Guilt, depressed, melancholy and disappointed exemplify Result-class terms falling close to the green sector.

In sum, emotions terms show the predicted azimuthal structure matching that of non-emotional process-stage prototypes shown in Figure 4. This matching is explained by process-semantic origin of emotions, implicitly reflected in word2vec data and revealed by the algorithm described in Section 4.2. Figure 5 thereby confirms theoretic hypothesis tested in the experiment.

\subsubsection{Purity of emotional states}

In the vertical view of the Bloch sphere shown in Figure 5, evaluation axis $\mathrm{Z}$ goes through the center of XY plane. Z coordinate of each term (17) is indicated by grayscale color of the marker. As prescribed by normalization All terms fall within the unitradius Bloch sphere as described by mixed-state vectors (7).

Proximity to the surface, measured by purity of qubit-emotional states, varies from curiosity $(x=-0.50, y=-0.73, z=-0.06)$ and misery $(x=0.38, y=0.39$, $z=-0.68)$ to interest $(x=-0.05, y=0, z=0.08)$. Near-origin position of interest, for example, indicates that this term is used uniformly across different process-class contexts.

In general, the more context-depending is meaning of emotion word (as explicated e.g. for disgust in S. Lee and Ellsworth (2013)), the more mixed is its qubit representation. Curiosity and misery, in contrast, definitely belong to Novelty and Result process stages, exemplifying most pure emotions of the studied set. Compared to randomly chosen word from e.g. Bradley and Lang (2017), emotion terms on average have higher purity and bear more process information contributing to their affective quality.

\subsection{Comparison with previously reported spaces of emotional concepts}

This section considers two emotional spaces reported by Morgan and Heise (1988) and Shaver, Schwartz, Kirson, and O'Connor (1987), constructed by multidimensional scaling of emotion-related judgments. Both spaces include three dimensions of Evaluation, Potency, and Activity, in which positions of emotional terms are provided as coordinate data. Among these dimensions, Potency and Activity span equatorial plane of the qubit semantic space in Bloch picture. Location of emotional terms in this plane is therefore expected to agree with the map shown in Figure 5. In particular, Fear-Surprise, Anger-Passion, and Sadness-Joy emotion classes should occupy distinct sectors of the azimuthal plane with minimal overlap with each other. This expectation 
a

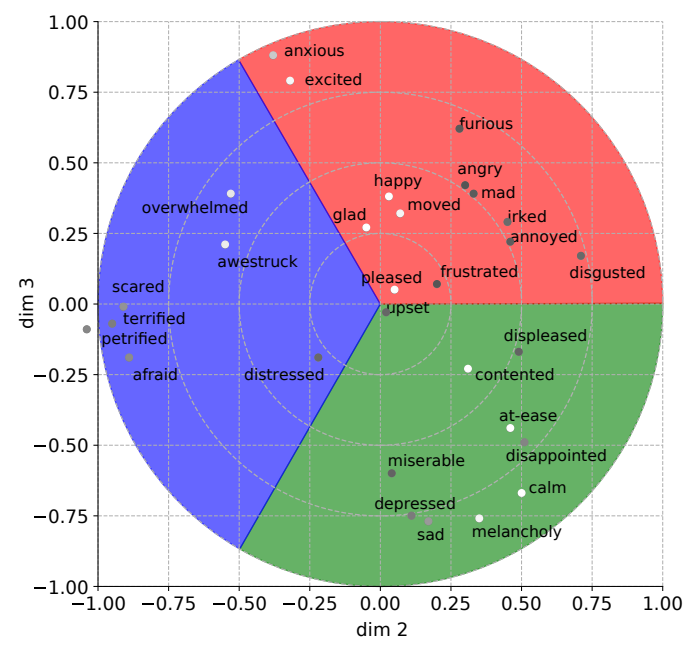

b

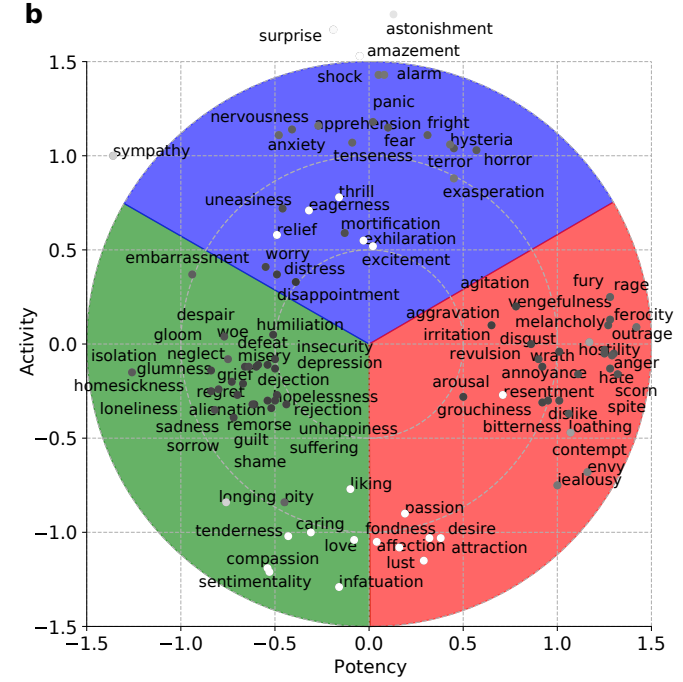

Figure 6. Position of emotion terms in Potency-Activity plane of three-dimensional space constructed by Morf (2018) (a) and Shaver et al. (1987) (b). Evaluation of each term is shown by color of the dot marker, with white and black corresponding to the maximal and minimal values. Novelty, Action, and Result sectors of the plane are shown in blue, red, and green.

is tested with two sets of data indicated above.

\subsubsection{Morgan and Heise (1988)}

Table 3 of Morgan and Heise (1988) provides location of 30 emotional terms in three Cartesian dimensions, among which second and third are interpreted as Potency and Activity. Positions of terms in this plane are shown in Figure 6(a). Circular structure of blue, red, and green $120^{\circ}$-sectors, denoting Novelty, Action, and Result process stages as before, is rotated by $60^{\circ}$ clockwise to maximize agreement with the expected affiliation of emotional terms.

The achieved agreement is obviously good. Namely, fear (afraid, petrified, terrified, scared) and surprise (awestruck, overwhelmed) terms are grouped in the Novelty $120^{\circ}$-range of $\phi$ shown in blue; anger (angry, furious, madness, irked, annoyed, disgusted) and passion (happy, moved) terms group in the red Action sector. Border with the blue sector locates near anxious-excited pair. Joy (contented, ease, calm) and sadness (sad, miserable, depressed, displeased, disappointed, melancholy) terms fall in the remaining green Result sector.

The only considerable deviation is glad term, expected in green instead of red sector. Distressed, frustrated, and pleased terms, also expected in green region, fall near its borders. This error could be minimized by adjustment of azimuthal width of sectors, here fixed to the baseline symmetric configuration. Azimuthal position of pleased and upset terms is poorly defined due to proximity to the origin.

\subsubsection{Shaver et al. (1987)}

Table 2 in Shaver et al. (1987) provides coordinates for 135 emotional terms in Evaluation-Potency-Activity space, also constructed by multidimensional scaling. Figure 4(b) shows 97 of these terms in Potency-Activity plane, located further than 0.5 
from the origin. Sector structure is rotated by $150^{\circ}$ clockwise in the same logic as for panel (a). In contrast to panel (a), the resulting location of sectors is mainly defined by negative-valued emotion terms forming distinct Fear, Anger, and Sadness clusters. Positive emotions from Joy and Zeal classes concentrate in a single cluster located at the border between red and green sectors. Except for several instances (e.g. sympathy, relief, exasperation, exhilaration), the resulting layout agrees with the expectation.

\subsubsection{Interpretation of the axes}

Figure 4 indicates that dimensions denoted by identical labels can be interpreted differently. For example, Activity ( $\operatorname{dim} 3$ ) of Fear-class terms in panel (a) is close to zero versus nearly maximal value in panel (b).

Process-semantic model of emotions differs from both of these views. As described in Section 3.3.1, fear accompanies detection of dangerous Novelty, requiring, first of all, recognition of the perceived signals involving memory and thinking. Archetypal freezing reflex provides time and physiological setting necessary for this essentially cognitive stage of intense internal activity. At the same time, external activity is low but growing as appropriate for the beginning of the process cycle. However quickly afterwards, fight and flight responses belong to the subsequent Action process stage accommodating observable actions ${ }^{3}$.

Vertical Y axis thus quantifies not intensity of activity as such. In fact, behavioral cycle does not have inactive stages, otherwise it would simply stop. What changes, however, is type of activity: from perception and cognition at Novelty stage, to observable behavior at Action, to feeling and reflection at the Result. In this logic expressed in Figures 3 and 5, Y axis quantifies externality of activity, discriminating internal from overt behavior.

\section{Quantum model of emotions in relation to other approaches}

This section summarizes relation of the developed theory and experimental results to the existing approaches to emotion modeling.

\subsection{Categorization of emotions}

Discretization of cognitive states is fundamental for natural cognition. It is required, in particular, by the same economic considerations that restrict natural thinking to pragmatic mode (Section 2.3). Economy dictates composition of cognitive dictionaries requiring energy for learning and use, as exemplified by regularities between functionality and usage of words in natural language Zipf (1945). Same holds for emotional categories Fehr and Russell (1984).

At emotional, pre-linguistic level of cognition (Section 5.6), dictionary of states is limited to 4-9 basic items Ekman (1992); Fehr and Russell (1984); Ortony and Turner (1990). This number is close to normal capacity of human attention, able to handle no more than $7 \pm 2$ objects simultaneously Miller (1956); fitting to this restriction, reasonable for achieving robust communication and behavioral control,

${ }^{3}$ Causation of fight-flight response by fear expresses ordering of the corresponding Action and Novelty stages in the process cycle, establishing their (probabilistic) causal relation, cf. Scarantino (2017a). 
explains baseline granularity of emotions Barrett (2004); J. Y. Lee, Lindquist, and Nam (2015) and parent / derivative concepts considered further in this section. In accord with Section 2.1, basic emotions can be seen as subjectively-recognized environmental (macro)states, used as optimal predictors of the future at various levels of biological hierarchy Flack (2017).

\subsubsection{Basic emotions}

Number of main emotion classes, defined in Section 3.3.1 as fear, surprise, zeal, rage, joy, and sadness), agrees with the restrictions just noted. This set also agrees with the results obtained from various research perspectives in composition. In particular, these classes are consistently derived from studies of situation classes, relational themes, neural activation patters, facial expressions, capacity of human attention, structure of our commitments and motives.

First, the considered taxonomy agrees with the models viewing emotions as distinct psycho-physiological scripts regulating behavior in archetypal situation classes covering activity of an organism Ekman (1992, 1999); Izard (1977); Johnson-Laird and Oatley (1992); Lucas (2018); Scarantino (2015); Tomkins (2008); the present model contributes to this picture by recognizing these classes, alternatively called core person-environment relational themes Lazarus (1991), as stages of a basic process cycle organizing subjective context representation.

Six components of human motivation including affiliation, power achievement, personal growth, altruism, stress avoidance, and sensation seeking Novacek and Lazarus (1990), sort to the basic process-semantic classes Novelty, Action, and Result abbreviated by first letters as R, A, N, R, N, A, respectively.

Primary affects of interest-excitement, surprise-startle, fear-terror, anger-rage, contempt-disgust, enjoyment-joy, distress-anguish, and shame-humiliation fall to three classes distinguished by their activation quality: the first triple of affects is activating, the next two maintaining activity at high level, while the last triple is inhibitory in nature Tomkins and Mccarter (1964). This classification, rooted in the density of neural firing, is in exact agreement with the process-semantic model in which activation occurs at Novelty stage, intense activity is maintained during Action, whereas slowdown accompanies the Result stage.

Baseline emotional categorization is refined by methods of hierarchical clustering. In agreement with the above, hierarchical level most useful for discrimination of emotions includes clusters love, joy/contentment, anger, sadness, fear, while sixth surprise class, although present at the same level, is less differentiated Keltner et al. (2019); Shaver et al. (1987); Storm and Storm (1987). Next hierarchical level includes up to 30 emotional classes Cowen and Keltner (2020); J. R. J. Fontaine, Poortinga, Setiadi, and Markam (2002); Horikawa, Cowen, Keltner, and Kamitani (2020); Shaver et al. (1987); Storm and Storm (1987). As for the basic four-to-nine, this number is not to be confused with dimensionality of an underlying space, as even a single dimension can accommodate unlimited number of classes. Instead, it indicates refined granularity of emotional space facilitating behavioral control and decision making Gendron and Barrett (2019); Lindquist and Barrett (2014). 


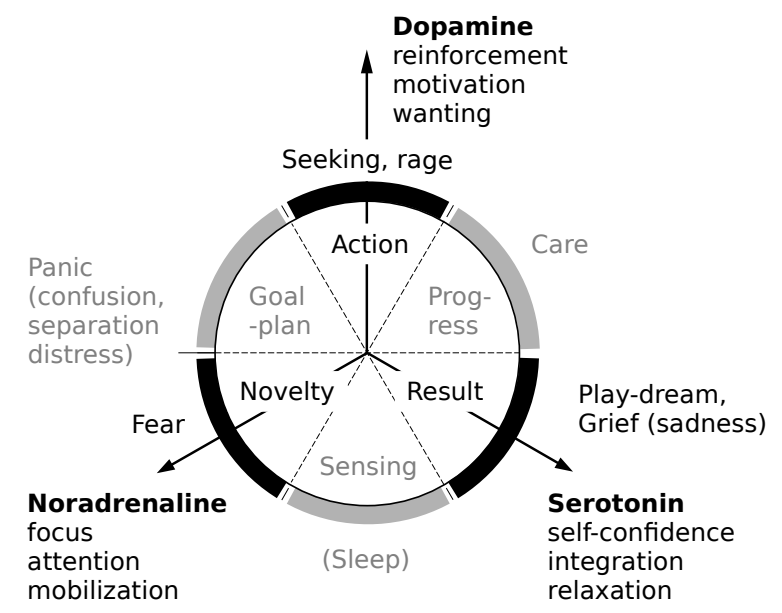

Figure 7. Neuro-physiological affective systems (fear, seeking, rage, care, play, panic-grief) and three monoamine neurotransmitters (noradrenaline, dopamine, serotonin) fundamental to working of mammalian brains Panksepp and Biven (2012) on the process-stage map of the qubit semantic space.

\subsubsection{Positive-negative asymmetry}

In practice, positive-negative symmetry evident in Figure 3 is broken by higher importance of negative emotions, so that lower Bloch-emotional hemisphere is resolved finer than the upper. Of the symmetric six, most suppressed is surprise category since archetypal evaluation of Novelty is clearly negative (Section 3.3). Remaining positive classes are often merged to a single happiness-joy class J. R. J. Fontaine et al. (2002); Jack, Sun, Delis, Garrod, and Schyns (2016), as shown e.g. in Figure 6(b). Vacant distinction slots can be occupied by more practical categories of hate, disgust, shame, and others Diener, Smith, and Fujita (1995); Ekman (1984); Oatley and Johnson-Laird (1987).

\subsection{Neurophysiological mechanics}

Physiological basis of process-semantic classes of emotions is identified in working of human brain. This section outlines two pieces of evidence.

\subsubsection{Panksepp's affective systems}

Basic affective systems of mammalian brains identified in Panksepp (2011) map to the azimuthal dimension of the qubit semantic space as shown in Figure 7. Namely, fear system processes Novelty; rage and seeking systems specialize on different kinds of Goal-plan and Action, with lust being combination of the two in specific context; care stands for feedback-associated Process stage, while play-dream is appropriate at the Result stage. Negative-valence panic-grief system seems to be ambivalent, processing both Goal-plan (panic, confusion, separation distress) and Result stages (sadness and grief).

\subsubsection{Functionality of basic neurotransmitters}

Specialization of affective systems parallels functionality of basic monoamine neurotransmitters, regulating neuronal activity within core system of animal's behavioral 
control Lövheim (2012); Panksepp (1998); Steinberg et al. (2013). Noradrenaline, dopamine, and serotonin correspond to the main triple of process stages as shown by arrows in Figure $7^{4}$. In particular, noradrenaline mobilizes the system and activates cognitive functions, necessary at Novelty and Goal-plan stages; dopamine, in turn, provides motivation and reinforcement propelling an organism for Action leading to the expected outcome; and serotonin, finally, facilitates slowdown and relaxation at the Result stage. These basic substances thus constitute chemical hardware of the process-semantic triad in human cognition Surov (2021a).

\subsection{Dimensionality of emotion space}

Dimensionality of the qubit-emotion space agrees with dimensionality of affective cognition identified by several approaches.

\subsubsection{Classical EPA dimensions}

$\mathrm{X}, \mathrm{Y}$, and $\mathrm{Z}$ axes of qubit semantic space described in Section 3.2.2 are close to classical dimensions of human affect called Potency (strain-relaxation, dominancesubmissiveness), and Activity (arousal), and Evaluation (pleasantness-unpleasantness) Mehrabian and Russell (1977); Osgood (1962); Schlosberg (1954); Wundt (1897). These dimensions are routinely considered as cartesian coordinates of threedimensional space, with Euclidean distance between points quantifying similarity of emotion states $^{5}$. Via trace distance between qubits (8) equivalent to Euclidean distance in Bloch representation, this establishes correspondence between classical EPA and qubit semantic spaces. The present model thereby gets related to classical semantic differential that appears as context-averaged limit of contextually-dependent semantics accounted by quantum approach Surov (2021b).

\subsubsection{Direct EPA measurement}

Work Morgan and Heise (1988) considered in Section 4.4.1 is unique in reporting EPA ratings of emotions terms, obtained via direct semantic-differential measurement, along with their multidimensional scaling solution. In contrast to the latter, directly measured ratings are highly correlated, with e.g. $85 \%$ of potency variation predictable from evaluation and activity scores. This essentially reduces three affective dimensions to two, thereby collapsing the process-semantic plane. Manifested in correlation between evaluation and potency, this problem is observed in other directly measured affective norms of English language Bradley and Lang (2017); Doyle and Bottomley (2010); Warriner, Kuperman, and Brysbaert (2013), with exception for Heise (1965).

Resolution of evaluation-neutral process-semantic structure of emotions can be maintained in two ways. The first involves full-fledged multidimensional scaling as e.g. in works considered in Section 4.4. Alternatively, fixing of the direct-measurement approach would require more accurate semantic differential scales, supplemented by methodological modifications further discussed in Section 5.5.2.

\footnotetext{
${ }^{4}$ Neurotransmitters usually function in combination with each other as well as with other hormones, forming complex chemical chords specialized for each affective mode Brown, Basheer, McKenna, Strecker, and McCarley (2012); Dayan (2012); Panksepp and Biven (2012). However, their individual main-theme semantics is clear Lövheim (2012).

${ }^{5}$ Alternatively, evaluation, potency, and activity can be represented as spherical coordinates in a fourdimensional hypersphere Sokolov and Boucsein (2000); Vartanov and Vartanova (2018). With nearly ideal shpericity, the resulting manifold is still three-dimensional.
} 


\subsubsection{Higher dimensions}

Human thinking is not limited to affective semantics covered by EPA space; as illustrated e.g. by word2vec and LSA spaces Landauer and Dumais (1997); Mikolov et al. (2013), regularities of symbolic cognition take no less than 100 dimensions of representation space. In emotion-metric experiments, they manifest as additional variance of judgment statistics unexplained by evaluation, potency, and activity. These "nonaffective factors" Russell (1980) can be accounted by supplementing EPA space with additional dimensions. Fourth dimension, for example, typically allows to capture additional 5-10 percent of total data variance Bush (1973); J. R. Fontaine, Scherer, Roesch, and Ellsworth (2007); Osgood (1962).

Non-obvious interpretation of fourth and higher dimensions (certainty, novelty, abstractness, neutrality, attraction-rejection) supports an essential difference between pre-conceptual and language-symbolic levels of cognition Gärdenfors (1995). The former, explained by universally interpretable EPA dimensions, can be identified as affective cognition ubiquitous in living nature Peil (2014). Language-symbolic, human-level cognition, in contrast, is modeled by high-dimensional, non-interpretable spaces exemplified by word2vec and LSA. Experimental method described in Section 4 essentially suggests mapping between these two types of representations.

\subsection{Circumplex and emotion wheel}

\subsubsection{Circumplex models}

Lower importance of third semantic factor compared to the first two in some cases allows to consider emotion concepts in two-dimensional space spanning evaluation and either activity or potency axes. Similarity judgments also suggest circular layout of emotions in the resulting plane, essentially reducing emotion space to a single curvilinear dimension Russell (1980); Schlosberg (1952).

Circular models of emotions are marginal case of the qubit-emotional space described in this work. In particular, valence-arousal circumplex Hevner (1936); Russell (1980) is obtained as section of Bloch-emotional sphere by YZ plane, shown in Figure 1 as bold circle. Namely, extreme pleasure and displeasure correspond to the poles 1 and 0 denoting assured realization of the basis decision alternatives, while arousal and sleepiness correspond to Action and Sensing process stages. In projection to this plane, discrimination of emotions in potency ( $\mathrm{X}$ axis, dominance in Mehrabian and Russell (1977)) is ignored, cf. Schacht (2013). As seen from Figure 3, joy then overlaps with inspiration, boredom with sadness, fear with depression, enthusiasm with acceptance.

Pleasantness/unpleasantness - attention/rejection emotional circle of Schlosberg (1952) corresponds to another vertical plane passing through $\sim 60^{\circ}$ and $\sim 240^{\circ}$ of azimuthal plane, corresponding to attention/rejection polarity. Six azimuthal classes fear-suffering, surprise, love-mirth-happy, anger-determination, contempt, disgust are then obtained by selective projection of the emotional sphere to this plane. Valence-power circumplex of Scherer, Shuman, Fontaine, and Soriano (2013) is understood in analogous way.

Cited models can be considered as empirically discovered, two-dimensional versions of the full qubit state space shown in Figure 3. Even when limited to pure emotional states located only at the surface, this model allows for filled circle of emotion locations in any projection, as observed e.g. in Schlosberg (1952) and Bush (1973). Besides, it 
allows for non-pure cognitive-emotional states describing subjective representation of uncontrolled, partially resolved, or underdefined behavioral alternatives accounted by mixed states (Section 3.1.2).

\subsubsection{Circumplex-based qubit models}

Several models establish partially interpretable qubit-emotional space by identifying valence-arousal circumplex with some section of the Bloch sphere. In particular, Yan et al. (2015) map pleasure (Evaluation) and arousal (Activity) affective dimensions to equatorial plane of the Bloch sphere, with polar $\mathrm{Z}$ axis quantifying intensity of emotions. Alternatively, pleasure is associated with $\mathrm{Z}$ axis Yan, Iliyasu, and Hirota (2021a), making the model equivalent to $\mathrm{ZY}$ plane of the Bloch sphere shown in Figure 3. Similar two-dimensional scheme is used to model collective emotions Tsarev, Trofimova, Alodjants, and Khrennikov (2019).

\subsubsection{Plutchik's model}

Plutchik's emotion solid is close to the present model in extending map of emotional states to three dimensions arranged in half-sphere structure Plutchik (1958). Although superficially similar, organization of emotions within this space differs. The main contrast is location of main emotions in circular dimension. With shaky logic behind it, Plutchik's ordering (disgust - expectancy - anger - joy - acceptance - surprise - fear - sadness) has little agreement with experiment Plutchik (1980). In process-semantic approach, this is explained by disparate nature of four basic opposites Kellerman (2020) underlying this order. In particular, joy-sadness and acceptance-disgust polarities as valence-wise within the same process-stage classes, while anger-fear and expectancy-surprise differ in process phase rather than in valence. Accordingly, qubit representation distinguishes these states in different spherical coordinates instead of a single circular dimension.

\subsection{Psychological scales as quantum observables}

\subsubsection{Parallel with physics}

Integration of diverse psychological dimensions achieved by valence-arousal circumplex M. Yik, Russell, and Steiger (2011); M. S. M. Yik, Russell, and Barrett (1999) exactly parallels the way in which qubit structure operates in physical models. Simplest example is Stern-Gerlach experiment in which individual electrons, entering external magnetic field, select to deviate either along or opposite to it. These two paths form basis states $|1\rangle,|0\rangle$ of the corresponding Hilbert space. In the context represented by state (1), electron chooses these alternatives with probabilities (3) (Feynman, Leyton, \& Sands, 1964, ch.6).

All possible experiments of that kind, differing by orientations of the magnets creating the magnetic field, are modeled in the same Hilbert space by rotating Bloch sphere's diameter defining alternative outcomes as shown in Figure 1. For example, apparatus with the field initially oriented at $\theta=0^{\circ}$ is said to measure " $\mathrm{Z}$ observable", while that with $\theta=\phi=90^{\circ}$ would measure "X observable" of the quantum state. Emotional variables mapped to different position in valence-arousal circumplex are psychological observables functioning in exactly the same way (Section 3.1.3). Harmonic correlation pattern between psychological scales reported in M. Yik et al. (2011) 
is typical correlation between two qubit observables as a function of angle $\alpha$ between them (10).

\subsubsection{Methodology of measurement}

Modeling of psychological scales as observables for subject's qubit-emotional state allows to analyze methods of emotion research from quantum-theoretical perspective. For example, evaluation, activity, and potency observables are immediately recognized as non-commuting. They require incompatible experimental setups (assignment phrasings) and therefore cannot be measured simultaneously ${ }^{6}$. When performed in succession, each observation modifies (cognitive-emotional) state of the system thereby affecting the following measurements White, Pothos, and Busemeyer (2014). The resulting "order effect" is one of several cognitive fallacies naturally modeled in quantum approach Busemeyer, Pothos, Franco, and Trueblood (2011); Pothos and Busemeyer (2013).

Non-commutativity of qubit observables exemplifies the proverbial measurement problem arising due to potentiality-actuality transition occurring in observation of quantum states: instead of passive copying of already existing information from system to meter, quantum "measurement" selects one of potential alternatives driving the system into a new state Gabora and Aerts (2005), (Busemeyer \& Bruza, 2012, ch. 1.1.2), (Jaeger, 2014, ch.3).

Actively-creative nature of quantum "measurement" implies changes in methodology of cognitive research Surov (2021a), applying to methods of emotion science as well. Estimation of evaluation, potency, and activity for multiple emotional terms by the same subject Bradley and Lang (2017); Doyle and Bottomley (2010); Warriner et al. (2013), for example, is obviously prone to incompatibility and "measurement" problems; corresponding order-type effects possibly explain drawbacks of this method mentioned in Section 5.3.2. This problem is (expensively) solved by using as much subjects as there are individual experiments; alternatively, one needs a technique for re-preparing subjects after their original states are altered by decision making. Further ideas can be borrowed from tomographic (quantum state estimation) methods developed in physics Řeháček, Englert, and Kaszlikowski (2004).

\subsection{Core affect and central assembly}

The proposed model of emotions is closely related to notions of central assembly Tomkins (1981) and core affect Russell and Barrett (1999) that can be seen as its conceptual precursors. According to Section 3, quantum version of core affect is defined as follows:

Quantum core affect is qubit state (1) encoding subjective representation of all available information relative to a particular two-way behavioral alternative resolved by the subject.

Analogous to quantum states of elementary physical systems, quantum core affect is potential state of an individual, unavailable for (or protected from) direct observation ${ }^{7}$. It encodes subjective intent, semantic disposition of an organism toward the basis

\footnotetext{
${ }^{6}$ The same non-commutativity effect for position and momentum observables of a massive particle is quantified by Heizenberg's uncertainty relation Feynman et al. (1964).

${ }^{7}$ Delineation between potential and actual, system and environment, known in physics as Heisenberg's cut, is crucial for quantum modeling Jaeger (2017); Surov (2021a). Via psycho-physiological parallelism Surov et
} 
decision alternative in a given context, fundamentally different from objectified "viewfrom-nowhere" description of the world Grush (1997); Nagel (1986); Surov (2021b).

Affective potentiality collapses with resolution of the basis uncertainty e.g. in the result of cognitive assessment Chen (2016); knowledge about the reason eliminates emotion, making emotionality and rational cognition complementary functions of mind (Solomon, 2003, ch.1). In physics this collapse is known as reduction of the wavefunction as a result of experiment-observation, evidenced e.g. in destroying interference in famous two-slit experiment Feynman et al. (1964). Quantum-theoretic description of these two examples is the same: what happens is collapse of the potentiality (Hilbert) space due to actualization of one of the basis alternatives previously "coexisting" in superposition potentiality state Surov (2021a).

\subsubsection{Core affect and psychological construction}

Classical core affect is defined as neurophysiological state, potentially accessible to consciousness as a primitive, non-reflective emotion Barrett and Bliss-Moreau (2009); Russell (2009); Russell and Barrett (1999). Essentially, it is compressed form of sensory data that "need not be directed at anything"; this view is properly expressed by "homeostatic barometer" metaphor, since pressure is scalar in nature. Subjective meaning of core affect, including emotional experience, is constructed by categorizing sensory input based on conceptual knowledge of an organism Barrett (2015a).

Expressions (1)-(2), in contrast, indicate that quantum core affect is vectorial by definition ${ }^{8}$. Since qubit state is constructed relative to a particular behavioral "difference that makes a (subjective) difference" (Section 3.2), quantum core affect is semantic from the start. In agreement with Gardenfors (2000), quantum semantics is therefore (pre-)conceptual in nature; symbolic-level cognition including natural language, however, may be used to reflect, communicate, and manipulate this subjective semantic state. Without symbolic representation, quantum core affect is still meaningful (i), and can be experienced as raw nonreflective emotion (ii). Classical core affect agrees with (ii), but seems to disagree with (i).

More precisely, quantum core affect represents (pre-)conceptual, subconscious level of cognition operating between unconscious sensory and conscious language-symbolic processing stages Gärdenfors $(1995)^{9}$. In agreement with classical account, this borderline between sensory and conceptual domains of cognition considered as a meaningmaking facility of an organism Lindquist, Wager, Kober, Bliss-Moreau, and Barrett (2012).

\subsubsection{Affective relativity}

Principal departure from the classical core affect, involved in the difference just mentioned, starts at "relative to" in the definition above. This introduces relativity feature inherent to quantum semantics Surov (2021b) and absent in other representation accounts of emotions Barrett and Fossum (2001); Khrennikov (2021); Pauen (2006);

al. (2021), this cut goes through both descriptions, ignoring Cartesian division between body and mind, cf. Atmanspacher (1997).

${ }^{8}$ More precisely, qubit representation contains both vector and scalar parts illustrated Figure 2.

${ }^{9}$ Anatomically, subconscious, pre-conceptual level of cognition corresponds to the limbic system sandwiched between reptilian and neocortex parts of the brain MacLEAN (1967); MacLean (1994). Relying on psychophysiological parallelism consistent with quantum modeling Surov et al. (2021), quantum core affect is defined in purely cognitive-informational "software-level" terms, for specifying its physiological carriers is object for parallel field of research. 
Reisenzein (2009); Shaver et al. (1987); Solomon (2003).

In quantum perspective, a person does not have an emotion just about a road accident that crashed his car; he is angry about disruption of his plans. In particular, this could be an alternative of making (1) a trip planned for the next weekend, or not (0). Only when this reason is not consciously recognized, emotion appears as "freefloating", or associated with the event, fact, or object itself. In practice, however, there are usually many decision alternatives affected by any given event. Along with anger for interfering with the trip, subject possibly fears to be found guilty for damage, and also enjoys staying alive, after all. Different core affects, possibly experienced as emotions, co-exist in distinct subjective Hilbert spaces generated for each basis alternative, represented by pure qubit states (1).

If different bases are not discriminated, corresponding core affects combine in a mixed state (5) of reduced purity. As described in Section 4.3.2, this is the case for word2vec representations averaged over large number of particular usages of concepts. By considering global neurophysiological state of an organism, classical core affect accounts for this average of multiple quantum core affects.

\subsubsection{Central assembly}

Quantum core affect is also close to the Tomkins' notion of central assembly, considered as principal function of affect Tomkins (1981). According to Tomkins, affect is universal motivational mechanism, driving realization of cognitive-behavioral tasks by amplifying their subjective urgency; like no instrument would work without power supply, no activity is possible without co-assembly with someone's affective system. Categorization of target cognitive-behavioral tasks then defines categorization of corresponding affects, experienced as eight or nine primary classes mentioned in Section 5.1.1.

By considering emotion as targeting particular tasks, Tomkins' theory subscribes for affective relativity. Crucial ingredient added to this by quantum approach is that infinite variety of tasks, targeted by affect, is reduced to a single elementary act of binary decision (Section 3.1). Quantum model thus achieves standard representation of this task requiring slow, deliberate thinking Kahneman (2011), cf. Hoorn and Ho (2019); after such decision is made, execution of a chosen scenario proceeds in energetically cheaper automatic mode Wood, Quinn, and Kashy (2002).

\section{Outlook: synergy of emotion science and quantum theory}

\subsection{Theoretical perspective}

Quantum approach offers simple conceptual system, in which notions of emotion science find intuitive mathematical expression. Crucially, this theory is not constructed ad-hoc, but follows from a single idea that emotions are subjective process-based representations of an organism's environment. The resulting model is nearly identical to quantum process-semantic model of context representation Surov (2021b), with mathematical part imported from standard quantum-theoretic formalism.

Agreement with fundamental features and models of emotion is then achieved automatically, illustrating conceptual affinity between psychological and quantum phenomena, already used for cognitive modeling Khrennikov (2015). This allows to consider quantum-theoretic approach as conceptual and mathematical framework sought for emotion studies Reisenzein (2019). Part of its potential for integration of affect with cognitive, brain, and behavioral sciences, envisioned by Alexandrov and Sams 
(2005); Anderson (1989); Barrett (2015b); Cornejo, Marsico, and Valsiner (2018); Dolan (2002); Duncan and Barrett (2007); Gintis (2007); Peil (2014); Pessoa (2019a); Roth and Jornet (2013); Woodward (2016), is shown in Sections 2 and 5.

\subsection{Practical perspective}

Modern informational technologies, including artificial intelligence, are growing without much of impact from emotion science. Central role of affect in human cognition Dukes et al. (2021); Duncan and Barrett (2007), however, would suggest the opposite. One reason why theories of emotion fall short of practical application is that they aim to model emotion itself, loosing connection to real-world problems requiring emotion-based solution (cf. Hoey et al. (2016)). The logic of natural development is, in contrast, practice-oriented: the very emotional system is developed by mammals not as a fanciful toy, but as a means to achieve advantage in socialization and behavioral control Goleman (2006).

Quantum approach tackles this problem by conceptualizing emotion in the broader scope of behavioral semantics. Contrary to the psychological studies encapsulated in the cognitive realm, it aims to model ordinary decision making practice Surov (2021a), cf. de Gelder (2017); George and Dane (2016); Lerner, Li, Valdesolo, and Kassam (2015). In that way, emotion science get natural connection to vast areas of applied science and technology. Couple of straightforward directions are outlined below.

\subsection{Quantum methods for practical emotion science}

Practice-oriented approach just mentioned considers emotions as subjective states defining probabilistic regularities of decision making ${ }^{10}$. Foundational steps in this direction are made in quantum models of cognition and decision developed in recent decades. In many cases, the correspondence is established simply by changing "cognitive" to "affective" state terminology. Then, applied emotion science is recognized in the existing models of cognitive "fallacies", "irrational" decision making, unexpected utility, non-classical economic behavior, semantics of natural language, information retrieval, and other Ashtiani and Azgomi (2015); Basieva, Khrennikova, Pothos, Asano, and Khrennikov (2018); Busemeyer et al. (2011); Haven and Khrennikov (2013); Khrennikov (2010, 2016); Melucci (2015); Moreira, Tiwari, Pandey, Bruza, and Wichert (2020); Njegovanovic (2018); Pothos and Busemeyer (2013). In their present state, these models, however, are far from realizing their full potential due to lack of understanding of the meaning of quantum-cognitive states. As further discussed below, methods of emotions science can become decisive factor bringing this approach to a new level of theoretical and practical impact.

Another prospect is illustrated by the method for studying emotional states based on databases of language semantics. Potential of this approach is evidenced by Tomkins' vision that natural languages are centuries ahead of psychology in modeling of subtle distinctions in affect complexes Tomkins (1981). Indeed, experiment of Section 4 showed that word2vec data reflect implicit affective information allowing for extraction

\footnotetext{
${ }^{10}$ This setting resonates with the goals pursued in robotics and AI Breazeal (2003); Cavallo et al. (2018); Deng, Huang, and Perkowski (2021); Kotov et al. (2021); Pessoa (2019b); Samsonovich (2020b); Vallverdú et al. (2016); Wolff et al. (2018); Yan, Iliyasu, and Hirota (2021b), (Samsonovich, 2020a, ch.7,10,13,34,54,56,57). As in natural cognitive systems, low-dimensional emotional states can be used to decouple stimulus from response Scherer (1982), as required for non-trivial decision making.
} 
and analysis. As other methods of language semantics in psychology and behavioral science Arnulf (2020); Arnulf, Larsen, Martinsen, and Bong (2014); Hollis and Westbury (2016); Jackson et al. (2019), this method suggests valuable complement for traditional experimental approaches in terms of scope, statistical reliability, and scalability.

\subsection{Emotion science for quantum cognition}

Currently, fundamental reasons underlying efficiency of quantum approach to cognitive and behavioral modeling are not widely agreed upon. Practice-oriented research therefore inclines to use quantum theory as "purely mathematical formalism, detached from any physical interpretation" - a sort of theoretical black box, producing correct probabilities for particular behavioral cases in rather mysterious way, cf. Blutner and beim Graben (2016); Pothos and Busemeyer (2013); Sozzo (2021). As a result, working models are often found by blind search with a lot of inefficient effort.

This paper envisions a way to solve this problem. Fully interpretable structure of the qubit Hilbert space suggests possibility for similar understanding of other quantumtheoretic notions. Concepts of emotional science could be useful to uncover psychological meaning, for example, of complex-valued probability amplitudes, interference phases, and irrationality factors, often treated as free fitting parameters with no definite meaning (cf. e.g. Favre, Wittwer, Heinimann, Yukalov, and Sornette (2016); Yukalov and Sornette (2010)). Estimation of these quantities by methods of emotion science, in turn, would allow to achieve predictive power requested for practical applications Surov, Pilkevich, Alodjants, and Khmelevsky (2019).

\section{Funding}

The research was supported by a grant of Russian Science Foundation (project number 20-71-00136).

\section{References}

Ackoff, R. L., \& Emery, F. E. (2017). On Purposeful Systems. An Interdisciplinary Analysis of Individual and Social Behavior as a System of Purposeful Events. Routledge.

Alexandrov, Y. I., \& Sams, M. E. (2005). Emotion and consciousness: Ends of a continuum. Cognitive Brain Research, 25(2), 387-405. Retrieved from https://linkinghub.elsevier.com/retrieve/pii/S0926641005002272

Anderson, N. H. (1989). Information integration approach to emotions and their measurement. In R. Plutchik \& H. Kellerman (Eds.), The measurement of emotions (chap. 6). New York: Academic Press.

Arnulf, J. K. (2020). Wittgenstein's Revenge: How Semantic Algorithms Can Help Survey Research Escape Smedslund's Labyrinth. In T. G. Lindstad, E. Stänicke, \& J. Valsiner (Eds.), Respect for thought (pp. 285-307). Springer.

Arnulf, J. K., Larsen, K. R., Martinsen, Ø. L., \& Bong, C. H. (2014). Predicting survey responses: How and why semantics shape survey statistics on Organizational Behaviour. PLoS ONE, 9(9).

Ashtiani, M., \& Azgomi, M. A. (2015). A survey of quantum-like approaches to decision making and cognition. Mathematical Social Sciences, 75, 49-80. Retrieved from http://dx.doi.org/10.1016/j.mathsocsci.2015.02.004 
Atmanspacher, H. (1997, sep). Cartesian cut, Heisenberg cut, and the concept of complexity. World Futures, 49(3), 333-355. Retrieved from http://www. tandfonline.com/doi/abs/10.1080/02604027.1997.9972639

Barrett, L. F. (2004). Feelings or words? Understanding the content in self-report ratings of experienced emotion. Journal of Personality and Social Psychology, 87(2), 266-281.

Barrett, L. F. (2015a). The conceptual act theory: A road map. In L. F. Barrett \& J. A. Russell (Eds.), The psychological construction of emotion (chap. 4). Guilford Press.

Barrett, L. F. (2015b). Construction as an integrative framework for the science of the emotion. In L. F. Barrett \& J. A. Russell (Eds.), The psychological construction of emotion (chap. 18). New York: Guilford Press.

Barrett, L. F. (2017, nov). The theory of constructed emotion: an active inference account of interoception and categorization. Social Cognitive and Affective Neuroscience, 12(11), 1833-1833. Retrieved from https://academic .oup.com/scan/article/12/11/1833/3788035

Barrett, L. F., \& Bliss-Moreau, E. (2009). Affect as a Psychological Primitive. In Advances in experimental social psychology (Vol. 41, pp. 167-218). Elsevier.

Barrett, L. F., \& Fossum, T. (2001). Mental representations of affect knowledge. Cognition and Emotion, 15(3), 333-363.

Barrett, L. F., \& Russell, J. A. (Eds.). (2015). The Psychological Construction of Emotion. Guilford Press.

Barrett, L. F., \& Simmons, W. K. (2015). Interoceptive predictions in the brain. Nature Reviews Neuroscience, 16(7), 419-429. Retrieved from http://dx.doi.org/10.1038/nrn3950

Basieva, I., Khrennikova, P., Pothos, E. M., Asano, M., \& Khrennikov, A. (2018). Quantumlike model of subjective expected utility. Journal of Mathematical Economics, 78, 150-162. Retrieved from https://doi.org/10.1016/j.jmateco.2018.02.001

Blutner, R., \& beim Graben, P. (2016). Quantum cognition and bounded rationality. Synthese, 193(10), 3239-3291.

Boiger, M., \& Mesquita, B. (2012, jul). The Construction of Emotion in Interactions, Relationships, and Cultures. Emotion Review, 4(3), 221-229. Retrieved from http://journals.sagepub.com/doi/10.1177/1754073912439765

Bradley, M. M., \& Lang, P. J. (2017). Affective Norms for English Words (ANEW): Instruction manual and affective ratings (Tech. Rep.). UF Center for the Study of Emotion and Attention, University of Florida.

Breazeal, C. (2003, jul). Emotion and sociable humanoid robots. International Journal of Human-Computer Studies, 59(1-2), 119-155. Retrieved from https://linkinghub.elsevier.com/retrieve/pii/S1071581903000181

Brown, R. E., Basheer, R., McKenna, J. T., Strecker, R. E., \& McCarley, R. W. (2012, jul). Control of Sleep and Wakefulness. Physiological Reviews, 92(3), 1087-1187.

Bubic, A., Yves von Cramon, D., \& Schubotz, R. I. (2010). Prediction, cognition and the brain. Frontiers in Human Neuroscience, 4(March), 1-15.

Busemeyer, J. R., \& Bruza, P. D. (2012). Quantum Models of Cognition and Decision. Cambridge University Press.

Busemeyer, J. R., Pothos, E. M., Franco, R., \& Trueblood, J. S. (2011). A quantum theoretical explanation for probability judgment errors. Psychological Review, 118(2), 193-218. Retrieved from http://doi.apa.org/getdoi.cfm?doi=10.1037/a0022542

Bush, L. E. (1973). Individual differences multidimensional scaling of adjectives denoting feelings. Journal of Personality and Social Psychology, 25(1), 50-57.

Carver, C. S., \& Scheier, M. F. (1990). Origins and functions of positive and negative affect: A control-process view. Psychological Review, 97(1), 19-35. Retrieved from http: //doi.apa.org/getdoi.cfm?doi=10.1037/0033-295X.97.1.19

Cavallo, F., Semeraro, F., Fiorini, L., Magyar, G., Sinčák, P., \& Dario, P. (2018, mar). Emotion Modelling for Social Robotics Applications: A Review. Journal of Bionic Engineering, 15(2), 185-203. Retrieved from 
http://link. springer.com/10.1007/s42235-018-0015-y

Chen, H. (2016). A Theoretic Review of Emotion Regulation. Open Journal of Social Sciences, $04(02), 147-153$.

Clark, A. (2013). Whatever next? Predictive brains, situated agents, and the future of cognitive science. Behavioral and Brain Sciences, 36(3), 181-204.

Cohen, J. D. (2005). The vulcanization of the human brain: A neural perspective on interactions between cognition and emotion. Journal of Economic Perspectives, 19(4), 3-24.

Conway, M. A., \& Bekerian, D. A. (1987). Situational knowledge and emotions. Cognition and Emotion, 1(2), 145-191.

Cornejo, C., Marsico, G., \& Valsiner, J. (2018). Conclusions: Affectivation as a return to vitality. In C. Cornejo \& J. Valsiner (Eds.), I activate you to affect me (chap. 18). Information Age Pblishing.

Corr, P. J. (2013, jun). Cold and hot cognition: Quantum probability theory and realistic psychological modeling. Behavioral and Brain Sciences, 36(3), 282-283.

Cowen, A. S., \& Keltner, D. (2020). Semantic Space Theory: A Computational Approach to Emotion. Trends in Cognitive Sciences, 113. Retrieved from https://doi.org/10.1016/j.tics.2020.11.004 https://linkinghub.elsevier.com/retrieve/pii/S136466132030276X

Dayan, P. (2012). Twenty-Five Lessons from Computational Neuromodulation. Neuron, $76(1), 240-256$. Retrieved from http://dx.doi.org/10.1016/j.neuron.2012.09.027

De Jesus, P. (2018). Thinking through enactive agency: sense-making, bio-semiosis and the ontologies of organismic worlds. Phenomenology and the Cognitive Sciences, 1-27. Retrieved from http://link.springer.com/10.1007/s11097-018-9562-2

de Gelder, B. (2017, jul). Going Native. Emotion Science in the TwentyFirst Century. Frontiers in Psychology, 8(JUL), 1-5. Retrieved from http://journal.frontiersin.org/article/10.3389/fpsyg. 2017.01212/full

Deng, R., Huang, Y., \& Perkowski, M. (2021, may). Quantum Motions and Emotions for a Humanoid Robot Actor. In 2021 ieee 51st international symposium on multiple-valued logic (ismvl) (pp. 207-214). IEEE. Retrieved from https : //ieeexplore.ieee.org/document/9459671/

Diener, E., Smith, H., \& Fujita, F. (1995). The Personality Structure of Affect. Journal of Personality and Social Psychology, 69(1), 130-141.

Dolan, R. J. (2002, nov). Emotion, Cognition, and Behavior. Science, 298(5596), 1191-1194. Retrieved from https://www.sciencemag.org/lookup/doi/10.1126/science.1076358

Doyle, J. R., \& Bottomley, P. A. (2010). Norms for Osgood's affective meaning (Evaluation, Potency, Activity): Ratings of Logos, Colors, Products and Services, Names, and Typefaces. SSRN Electronic Journal, 1-27.

Doyle McCarthy, E. (1994). The social construction of emotions: New directions from culture theory. Social Perspectives on Emotion, 2(24), 267279. Retrieved from http://faculty.fordham.edu/mccarthy/Social Construction of Emotions 1994.pdf

Dukes, D., Abrams, K., Adolphs, R., Ahmed, M. E., Beatty, A., Berridge, K. C., .. Sander, D. (2021, jul). The rise of affectivism. Nature Human Behaviour, 5(7), 816-820. Retrieved from http://www. nature.com/articles/s41562-021-01130-8

Duncan, S., \& Barrett, L. F. (2007). Affect is a form of cognition: A neurobiological analysis. Cognition and Emotion, 21 (6), 1184-1211.

Ekman, P. (1984). Expression and the nature of emotion. In K. R. Scherer \& P. Ekman (Eds.), Approaches to emotion (Vol. 3, p. 344).

Ekman, P. (1992, may). An argument for basic emotions. Cognition and Emotion, 6(3-4), 169-200. Retrieved from https://www.tandfonline.com/doi/full/10.1080/02699939208411068

Ekman, P. (1999). Basic Emotions. In T. Dalgleish \& M. Power (Eds.), Handbook of cognition and emotion (pp. 45-60). 
Favre, M., Wittwer, A., Heinimann, H. R., Yukalov, V. I., \& Sornette, D. (2016, dec). Quantum Decision Theory in Simple Risky Choices. PLOS ONE, 11(12), e0168045. Retrieved from https://dx.plos.org/10.1371/journal.pone.0168045

Fehr, B., \& Russell, J. A. (1984). Concept of emotion viewed from a prototype perspective. Journal of Experimental Psychology: General, 113(3), 464-486.

Feynman, R. P., Leyton, R. B., \& Sands, M. (1964). Feynman Lectures in Physics vol. III.

Fink, G., \& Yolles, M. (2018, jan). Affect and cognition, part 1: "crossfire" interaction model. Kybernetes, 47(1), 80-98. Retrieved from https://www . emerald.com/insight/content/doi/10.1108/K-07-2017-0262/full/html

Flack, J. (2017). Life's Information Hierarchy. In S. I. Walker, P. C. W. Davies, \& G. F. R. Ellis (Eds.), From matter to life (pp. 283-302). Cambridge: Cambridge University Press.

Fontaine, J. R., Scherer, K. R., Roesch, E. B., \& Ellsworth, P. C. (2007, dec). The World of Emotions is not Two-Dimensional. Psychological Science, 18(12), 1050-1057. Retrieved from http://journals.sagepub.com/doi/10.1111/j.1467-9280.2007.02024.x

Fontaine, J. R. J., Poortinga, Y. H., Setiadi, B., \& Markam, S. S. (2002). Cognitive structure of emotion terms in Indonesia and The Netherlands. Cognition and Emotion, 16(1), 61-86.

Gabora, L., \& Aerts, D. (2005, mar). Evolution as context-driven actualisation of potential: toward an interdisciplinary theory of change of state. Interdisciplinary Science Reviews, 30(1), 69-88. Retrieved from http://www.tandfonline.com/doi/full/10.1179/030801805X25873

Gärdenfors, P. (1995). Three levels of inductive inference. In Studies in logic and the foundations of mathematics (Vol. 134, pp. 427-449). Retrieved from https://linkinghub.elsevier.com/retrieve/pii/S0049237X06800558

Gardenfors, P. (2000). Conceptual Spaces. The Geometry of Thought. MIT Press. Retrieved from https://mitpress.mit.edu/books/conceptual-spaces

Gendron, M., \& Barrett, L. F. (2019). A Role for Emotional Granularity in Judging. Oñati Socio-legal Series, 9(9(5)), 557-576.

Gendron, M., Mesquita, B., \& Barrett, L. F. (2020). The Brain as a Cultural Artifact. Concepts, Actions, and Experiences within the Human Affective Niche. In Culture, mind, and brain (pp. 188-222). Cambridge University Press.

George, J. M., \& Dane, E. (2016). Affect, emotion, and decision making. Organizational Behavior and Human Decision Processes, 136, 47-55. Retrieved from http://dx.doi.org/10.1016/j.obhdp.2016.06.004

Gintis, H. (2007). A framework for the unification of the behavioral sciences. Behavioral and Brain Sciences, 30(1-61), 1-61.

Gładziejewski, P. (2016, feb). Predictive coding and representationalism. Synthese, 193(2), 559-582. Retrieved from http://link.springer.com/10.1007/s11229-015-0762-9

Goleman, D. (2006). Social Intelligence. The New Science of Human Relationships. Bantam books.

Google Code Archive. (2013). word2vec. Retrieved 2021-02-01, from https://code.google.com/archive/p/word2vec/

Greve, P. F. (2015). The role of prediction in mental processing: A process approach. New Ideas in Psychology, 39, 45-52. Retrieved from http://dx.doi.org/10.1016/j.newideapsych.2015.07.007

Gross, J. J. (2015, jan). Emotion Regulation: Current Status and Future Prospects. Psychological Inquiry, 26(1), 1-26. Retrieved from http://www.tandfonline.com/doi/abs/10.1080/1047840X . 2014.940781

Grush, R. (1997, mar). The architecture of representation. Philosophical Psychology, 10(1), 5-23. Retrieved from http: //www .tandfonline.com/doi/abs/10.1080/09515089708573201

Haven, E., \& Khrennikov, A. (2013). Quantum Social Science. New York: Cambridge University Press.

Heise, D. R. (1965). Semantic differential profiles for 1,000 most frequent English 
words. Psychological Monographs: General and Applied, 79(8), 1-31. Retrieved from http://doi.apa.org/getdoi.cfm?doi=10.1037/h0093884

Hevner, K. (1936). Experimental Studies of the Elements of Expression in Music. The American Journal of Psychology, 48(2), 246. Retrieved from https://www . jstor.org/stable/1415746

Hoey, J., Schröder, T., \& Alhothali, A. (2016). Affect control processes: Intelligent affective interaction using a partially observable Markov decision process. Artificial Intelligence, 230, 134-172. Retrieved from http://dx.doi.org/10.1016/j.artint.2015.09.004

Hollis, G., \& Westbury, C. (2016). The principals of meaning: Extracting semantic dimensions from co-occurrence models of semantics. Psychonomic Bulletin and Review, 23(6), 17441756. Retrieved from http://dx.doi.org/10.3758/s13423-016-1053-2

Hoorn, J. F., \& Ho, J. K. W. (2019). Robot Affect: the Amygdala as Bloch Sphere. Retrieved from http://arxiv.org/abs/1911.12128

Horikawa, T., Cowen, A. S., Keltner, D., \& Kamitani, Y. (2020). The Neural Representation of Visually Evoked Emotion Is High-Dimensional, Categorical, and Distributed across Transmodal Brain Regions. iScience, 23(5), 101060. Retrieved from https://doi.org/10.1016/j.isci.2020.101060

Izard, C. E. (1977). Human Emotions. Springer.

Jack, R. E., Sun, W., Delis, I., Garrod, O. G. B., \& Schyns, P. G. (2016). Four not six: Revealing culturally common facial expressions of emotion. Journal of Experimental Psychology: General, 145(6), 708-730.

Jackson, J. C., Watts, J., Henry, T. R., List, J.-m., Forkel, R., Mucha, P. J., ... Lindquist, K. A. (2019). Emotion semantics show both cultural variation and universal structure. Science, 366(6472), 1517-1522. Retrieved from https : //www.sciencemag.org/lookup/doi/10.1126/science. aaw8160

Jaeger, G. (2007). Quantum Information: An Overview. Springer.

Jaeger, G. (2014). Quantum Objects.

Jaeger, G. (2017). "Wave-packet reduction" and the quantum character of the actualization of potentia. Entropy, 19(10).

Johnson-Laird, P. N., \& Oatley, K. (1992). Basic Emotions, Rationality, and Folk Theory. Cognition and Emotion, 6(3-4), 201-223.

Kahneman, D. (2011). Thinking, Fast and Slow. New York: Farrar, Straus and Giroux.

Kellerman, H. (2020). The Unconscious Domain.

Kelly, G. A. (2003). The psychology of personal constructs, vol.1: A theory of personality. Routledge.

Keltner, D., Sauter, D., Tracy, J., \& Cowen, A. (2019). Emotional Expression: Advances in Basic Emotion Theory. Journal of Nonverbal Behavior, 43(2), 133-160. Retrieved from https : //doi .org/10.1007/s10919-019-00293-3

Khrennikov, A. (2010). Ubiquitous Quantum Structure. From psychology to finance. Berlin, Heidelberg: Springer Berlin Heidelberg. Retrieved from http: //link. springer. com/10.1007/978-3-642-05101-2

Khrennikov, A. (2015). Quantum-like modeling of cognition. Frontiers in Physics, 3(77), 77. Retrieved from http://journal.frontiersin.org/Article/10.3389/fphy.2015.00077/abstract

Khrennikov, A. (2016, jan). 'Social Laser': action amplification by stimulated emission of social energy. Philosophical Transactions of the Royal Society A: Mathematical, Physical and Engineering Sciences, 374(2058), 20150094. Retrieved from https://royalsocietypublishing.org/doi/10.1098/rsta.2015.0094

Khrennikov, A. (2021, oct). Quantum-like model for unconscious-conscious interaction and emotional coloring of perceptions and other conscious experiences. Biosystems, 208(May), 104471. Retrieved from https://linkinghub.elsevier.com/retrieve/pii/S0303264721001234

Kolchinsky, A., \& Wolpert, D. H. (2018). Semantic information, autonomous agency and non-equilibrium statistical physics. Interface Focus, 8(6), 20180041. Retrieved from 
http://www.royalsocietypublishing.org/doi/10.1098/rsfs.2018.0041

Kotov, A., Zaidelman, L., Zinina, A., Arinkin, N., Filatov, A., \& Kivva, K. (2021). Conceptual processing system for a companion robot. Cognitive Systems Research, 67, 28-32. Retrieved from https://doi.org/10.1016/j.cogsys.2020.12.007

Kuznetsov, N. A., Baksanskii, O. E., \& Zholkov, S. Y. (2012, aug). Sources and foundation of pragmatic knowledge. Journal of Communications Technology and Electronics, 57(8), 868-881. Retrieved from http://link.springer.com/10.1134/S1064226912080025

Landauer, T. K., \& Dumais, S. T. (1997). A Solution to Plato's Problem: The Latent Semantic Analysis Theory of Acquisition, Induction, and Representation of Knowledge. Psychological Review, 104(2), 211-240.

Lazarus, R. S. (1991). Progress on a cognitive-motivational-relational theory of emotion. American Psychologist, 46(8), 819-834.

Lazarus, R. S. (1995). Emotions Express a Social Relationship, but It Is an Individual Mind That Creates Them. Psychological Inquiry, 6(3), 253-265.

Le Bellac, M. (2006). A Short Introduction to Quantum Information and Quantum Computation. Cambridge University Press.

Lee, J. Y., Lindquist, K. A., \& Nam, C. S. (2015). Predicting emotional granularity with EEG coherence. Proceedings of the Human Factors and Ergonomics Society, 2015-Janua(June 2016), 120-124.

Lee, S., \& Ellsworth, P. (2013). Maggots and morals: Physical disgust is to fear as moral disgust is to anger. In J. R. J. Fontaine, K. Scherer, \& C. Soriano (Eds.), Components of emotional meaning (pp. 271-280). Oxford University Press. Retrieved from https://oxford.universitypressscholarship.com/view/10.1093/acprof : oso/9780199592746.001.0001/

Lerner, J. S., Li, Y., Valdesolo, P., \& Kassam, K. S. (2015). Emotion and decision making. Annual Review of Psychology, 66, 799-823.

Lindquist, K. A., \& Barrett, L. F. (2014). Emotional complexity. Handbook of Emotions(December), 513-530.

Lindquist, K. A., Wager, T. D., Kober, H., Bliss-Moreau, E., \& Barrett, L. F. (2012, jun). The brain basis of emotion: A meta-analytic review. Behavioral and Brain Sciences, 35(3), 121-143.

Lövheim, H. (2012). A new three-dimensional model for emotions and monoamine neurotransmitters. Medical Hypotheses, 78(2), 341-348.

Lucas, D. A. (2018). Affect Theory, Genre, and the Example of Tragedy.

MacLEAN, P. D. (1967). The brain in relation to empathy and medical education. The Journal of Nervous and Mental Disease, 144(5), 374-382. Retrieved from http://journals. Iww.com/00005053-196705000-00005

MacLean, P. D. (1994). Human Nature: Duality or Triality? Politics and the Life Sciences, $13(1), 107-112$.

Mehrabian, A., \& Russell, J. A. (1977). Evidence for a Three-Factor Theory of Emotions. Journal of Research in Personality, 11, 273-294.

Melucci, M. (2015). Introduction to Information Retrieval and Quantum Mechanics (Vol. 35). Berlin, Heidelberg: Springer Berlin Heidelberg. Retrieved from http: //link. springer.com/10.1007/978-3-662-48313-8

Mesquita, B. (2010). Emoting: A contextualized process. In B. Mesquita, L. F. Barrett, \& E. R. Smith (Eds.), The mind in context (pp. 83-104). The Guilford Press.

Mesquita, B., Barrett, L. F., \& Smith, E. R. (Eds.). (2010). The mind in context.

Mikolov, T., Chen, K., Corrado, G., \& Dean, J. (2013). Efficient estimation of word representations in vector space. arXiv:1301.3781.

Miller, G. A. (1956). The magical number seven, plus or minus two: some limits on our capacity for processing information. Psychological Review, 63(2), 81-97. Retrieved from http://doi .apa.org/getdoi.cfm?doi=10.1037/h0043158

Mobbs, D., Adolphs, R., Fanselow, M. S., Barrett, L. F., LeDoux, J. E., Ressler, K., \& Tye, K. M. (2019). Viewpoints: Approaches to defining and in- 
vestigating fear. Nature Neuroscience, 22(8), 1205-1216. Retrieved from http://dx.doi.org/10.1038/s41593-019-0456-6

Moors, A., \& Fischer, M. (2019). Demystifying the role of emotion in behaviour: toward a goal-directed account. Cognition and Emotion, 33(1), 94-100. Retrieved from https://doi .org/10.1080/02699931.2018.1510381

Moreira, C., Tiwari, P., Pandey, H. M., Bruza, P., \& Wichert, A. (2020). Quantum-like influence diagrams for decision-making. Neural Networks, 132, 190-210. Retrieved from https://doi.org/10.1016/j.neunet.2020.07.009

Morf, M. E. (2018). Agency, Chance, and the Scientific Status of Psychology. Integrative Psychological and Behavioral Science, 52(4), 491-507.

Morgan, R. L., \& Heise, D. (1988, mar). Structure of Emotions. Social Psychology Quarterly, $51(1), 19$. Retrieved from http://www.jstor.org/stable/2786981?origin=crossref

Nagel, T. (1986). The View From Nowhere. Oxford University Press.

Nielsen, M. A., \& Chuang, I. L. (2010). Quantum Computation and Quantum Information. Cambridge: Cambridge University Press. Retrieved from http: //ebooks . cambridge.org/ref/id/CB09780511976667

Njegovanovic, A. (2018). Hilbert Space / Quantum Theory of the Financial Decision and Role of the Prefrontal Cortex with a View to Emotions. International Journal of Social and Administrative Sciences, 3(1), 42-54. Retrieved from http://www. aessweb.com/journals/March2018/5051/4269

Novacek, J., \& Lazarus, R. S. (1990, dec). The Structure of Personal Commitments. Journal of Personality, 58(4), 693-715. Retrieved from http://doi.wiley.com/10.1111/j.1467-6494.1990.tb00250.x

Oatley, K., \& Johnson-Laird, P. N. (1987). Towards a Cognitive Theory of Emotions. Cognition and Emotion, 1(1), 29-50.

Ortony, A., \& Turner, T. J. (1990). What's basic about basic emotions? Psychological Review, $97(3), 315-331$.

Osgood, C. E. (1962, jan). Studies on the generality of affective meaning systems. American Psychologist, 17(1), 10-28. Retrieved from http://doi.apa.org/getdoi.cfm?doi=10.1037/h0045146

Panksepp, J. (1998). Affective Neuroscience: The Foundations of Human and Animal Emotions.

Panksepp, J. (2011). Cross-Species affective neuroscience decoding of the primal affective experiences of humans and related animals. PLoS ONE, 6 (9).

Panksepp, J., \& Biven, L. (2012). The archaeology of mind. Neuroevolutionary origins of human emotion. Norton and Company.

Pauen, M. (2006). Emotion, Decision, and Mental Models. In C. Held, M. Knauff, \& G. Vosgerau (Eds.), Mental models and the mind. current developments in cognitive psychology, neuroscience, and philosophy of mind (p. 173). Retrieved from http://library1.nida.ac.th/termpaper6/sd/2554/19755.pdf

Peil, K. T. (2014, mar). Emotion: the Self-regulatory Sense. Global Advances in Health and Medicine, 3(2), 80-108. Retrieved from http://journals.sagepub.com/doi/10.7453/gahmj.2013.058

Pessoa, L. (2019a). Embracing integration and complexity: placing emotion within a science of brain and behaviour. Cognition and Emotion, 33(1), 55-60. Retrieved from https://doi.org/10.1080/02699931.2018.1520079

Pessoa, L. (2019b). Intelligent architectures for robotics: The merging of cognition and emotion. Physics of Life Reviews, 31, 157-170. Retrieved from https://doi.org/10.1016/j.plrev.2019.04.009

Plutchik, R. (1958). Outlines of a New Theory of Emotion. Transactions of the New York Academy of Sciences, 20(5 Series II), 394-403.

Plutchik, R. (1980). A General Psychoevolutionary Theory of Emotion. In Theories of emotion (Vol. 1, pp. 3-33). Academic Press. Retrieved from http: //dx.doi .org/10.1016/B978-0-12-558701-3.50007-7 
Plutchik, R. (1983). Emotions in Early Development: a Psychoevolutionary Approach. In Emotions in early development (Vol. 2, pp. 221-257). Academic Press. Retrieved from http://dx.doi.org/10.1016/B978-0-12-558702-0.50014-5

Pothos, E. M., \& Busemeyer, J. R. (2013, jun). Can quantum probability provide a new direction for cognitive modeling? The Behavioral and brain sciences, 36(3), 255-74. Retrieved from http://www.ncbi.nlm.nih.gov/pubmed/23673021

Reisenzein, R. (2009, mar). Emotions as metarepresentational states of mind: Naturalizing the belief-desire theory of emotion. Cognitive Systems Research, 10(1), 6-20. Retrieved from https://linkinghub.elsevier.com/retrieve/pii/S1389041708000272

Reisenzein, R. (2019). Cognition and emotion: a plea for theory. Cognition and Emotion, 33(1), 109-118. Retrieved from https://doi.org/10.1080/02699931.2019.1568968

Roth, W. M., \& Jornet, A. (2013). Situated cognition. Wiley Interdisciplinary Reviews: Cognitive Science, 4(5), 463-478.

Russell, J. A. (1980). A circumplex model of affect. Journal of Personality and Social Psychology, 39(6), 1161-1178. Retrieved from http: //content .apa.org/journals/psp/39/6/1161

Russell, J. A. (2009). Emotion, core affect, and psychological construction. Cognition and Emotion, 23(7), 1259-1283.

Russell, J. A., \& Barrett, L. F. (1999). Core affect, prototypical emotional episodes, and other things called emotion: Dissecting the elephant. Journal of Personality and Social Psychology, 76(5), 805-819. Retrieved from http: //doi.apa.org/getdoi.cfm?doi=10.1037/0022-3514.76.5.805

Samsonovich, A. V. (Ed.). (2020a). Biologically Inspired Cognitive Architectures (Vol. 948). Cham: Springer International Publishing. Retrieved from http: //link. springer. com/10.1007/978-3-030-25719-4

Samsonovich, A. V. (2020b). Socially emotional brain-inspired cognitive architecture framework for artificial intelligence. Cognitive Systems Research, 60, 57-76. Retrieved from https://doi.org/10.1016/j.cogsys.2019.12.002

Scarantino, A. (2015). Basic emotions, Psychological construction, and the Problem of Variability. In L. F. Barrett \& J. A. Russell (Eds.), The psychological construction of emotion (pp. 334-376). Guilford Press.

Scarantino, A. (2017a). Do emotions cause actions, and if so how? Emotion Review, 9(4), $326-334$.

Scarantino, A. (2017b). How to Do Things with Emotional Expressions: The Theory of Affective Pragmatics. Psychological Inquiry, 28(2-3), 165-185. Retrieved from https : //doi .org/10.1080/1047840X.2017.1328951

Schacht, A. (2013). Reviving a Forgotten Dimension - Potency in Affective Neuroscience. In Components of emotional meaning.

Scherer, K. R. (1982). Emotion as a process: function, origin and regulation. Social Science Information, 4/5, 555-570.

Scherer, K. R., Shuman, V., Fontaine, J. R. J., \& Soriano, C. (2013, aug). The GRID meets the Wheel: Assessing emotional feeling via self-report1. In Components of emotional meaning (pp. 281-298). Oxford University Press. Retrieved from https: //oxford.universitypressscholarship.com/view/10.1093/acprof : oso/9780199592746.001.0001/

Schlosberg, H. (1952). The description of facial expressions in terms of two dimensions. Journal of Experimental Psychology, 44(4), 229-237.

Schlosberg, H. (1954). Three dimensions of emotion. Psychological Review, 61(2), 81-88. Retrieved from http://doi.apa.org/getdoi.cfm?doi=10.1037/h0054570

Shaver, P., Schwartz, J., Kirson, D., \& O’Connor, C. (1987). Emotion knowledge: Further exploration of a prototype approach. Journal of Personality and Social Psychology, 52(6), 1061-1086.

Sokolov, E. N., \& Boucsein, W. (2000). A psychophysiological model of emotion space. Integrative Physiological and Behavioral Science, 35(2), 81-119. Retrieved from 
http://link.springer.com/10.1007/BF02688770

Solomon, R. (2003). Not Passion's Slave: Emotions and Choice. Oxford University Press.

Sozzo, S. (2021). Representing Attitudes Towards Ambiguity in Hilbert Space: Foundations and Applications. Foundations of Science, 26(1), 103-128. Retrieved from https://doi.org/10.1007/s10699-020-09718-5

Steinberg, E. E., Keiflin, R., Boivin, J. R., Witten, I. B., Deisseroth, K., \& Janak, P. H. (2013, jul). A causal link between prediction errors, dopamine neurons and learning. Nature Neuroscience, 16(7), 966-973. Retrieved from http://www.nature.com/articles/nn.3413

Storm, C., \& Storm, T. (1987). A Taxonomic Study of the Vocabulary of Emotions. Journal of Personality and Social Psychology, 53(4), 805-816.

Surov, I. A. (2021a). Quantum Cognitive Triad: Semantic Geometry of Context Representation. Foundations of Science, 26(4), 947-975. Retrieved from https : //link. springer.com/10.1007/s10699-020-09712-x

Surov, I. A. (2021b). Quantum Process Semantics. Preprints. Retrieved from https://www.preprints.org/manuscript/202109.0006/v1

Surov, I. A., \& Alodjants, A. P. (2018). Models of decision making in quantum cognition (in Russian). Saint-Petersburg: ITMO University.

Surov, I. A., Pilkevich, S. V., Alodjants, A. P., \& Khmelevsky, S. V. (2019). Quantum Phase Stability in Human Cognition. Frontiers in Psychology, 10(April), 1-6. Retrieved from https://www.frontiersin.org/article/10.3389/fpsyg.2019.00929/full

Surov, I. A., Semenenko, E., Platonov, A. V., Bessmertny, I. A., Galofaro, F., Toffano, Z., ... Alodjants, A. P. (2021, dec). Quantum semantics of text perception. Scientific Reports, $11(1), 4193$. Retrieved from http://www .nature.com/articles/s41598-021-83490-9

Tarski, A. (1944). The Semantic Conception of Truth: and the Foundations of Semantics. Philosophy and Phenomenological Research, 4(3), 341.

Tomkins, S. S. (1981). The quest for primary motives: Biography and autobiography of an idea. Journal of Personality and Social Psychology, 41(2), 306-329. Retrieved from http: //content . apa.org/journals/psp/41/2/306

Tomkins, S. S. (2008). Affect Imagery Consciousness. Springer.

Tomkins, S. S., \& Mccarter, R. (1964). What and Where Are the Primary Affects? Some Evidence for a Theory. Perceptual and motor skills, 18, 119-158.

Tsarev, D., Trofimova, A., Alodjants, A., \& Khrennikov, A. (2019, dec). Phase transitions, collective emotions and decision-making problem in heterogeneous social systems. Scientific Reports, 9(1), 18039. Retrieved from http://www . nature.com/articles/s41598-019-54296-7

Vallverdú, J., Talanov, M., Distefano, S., Mazzara, M., Tchitchigin, A., \& Nurgaliev, I. (2016, jan). A cognitive architecture for the implementation of emotions in computing systems. Biologically Inspired Cognitive Architectures, 15, 34-40. Retrieved from https://linkinghub.elsevier.com/retrieve/pii/S2212683X15000663

Vartanov, A. V., \& Vartanova, I. I. (2018). Four-dimensional Spherical Model of Emotions. Procedia Computer Science, 145, 604-610. Retrieved from https://doi.org/10.1016/j.procs.2018.11.096

Warriner, A. B., Kuperman, V., \& Brysbaert, M. (2013). Norms of valence, arousal, and dominance for 13,915 English lemmas. Behavior Research Methods, 45(4), 1191-1207.

White, L. C., Pothos, E. M., \& Busemeyer, J. R. (2014). Sometimes it does hurt to ask: The constructive role of articulating impressions. Cognition, 133(1), 48-64. Retrieved from http://dx.doi.org/10.1016/j.cognition.2014.05.015

Wolff, M., Huber, M., Wirsching, G., Romer, R., Graben, P. B., \& Schmitt, I. (2018, aug). Towards a Quantum Mechanical Model of the Inner Stage of Cognitive Agents. In 2018 9th ieee international conference on cognitive infocommunications (coginfocom) (pp. 000147000152). IEEE. Retrieved from https://ieeexplore.ieee.org/document/8639892/

Wood, W., Quinn, J. M., \& Kashy, D. A. (2002). Habits in everyday life: Thought, emotion, and action. Journal of Personality and Social Psychology, 83(6), 1281-1297.

Woodward, J. (2016). Emotion Versus Cognition in Moral Decision-Making: A Dubious 
Dichotomy. In S. M. Liao (Ed.), Moral brains: the neuroscience of morality (pp. 87-116). Oxford University Press.

Wundt, W. M. (1897). Outlines of Psychology. Nalanda Digital Library. Retrieved from http://psychclassics.yorku.ca/Wundt/Outlines/index.htm

Yan, F., Iliyasu, A. M., \& Hirota, K. (2021a, jan). Conceptual Framework for Quantum Affective Computing and Its Use in Fusion of Multi-Robot Emotions. Electronics, 10(2), 100. Retrieved from https://www.mdpi.com/2079-9292/10/2/100

Yan, F., Iliyasu, A. M., \& Hirota, K. (2021b). Emotion space modelling for social robots. Engineering Applications of Artificial Intelligence, 100(February), 104178. Retrieved from https://doi.org/10.1016/j.engappai.2021.104178

Yan, F., Iliyasu, A. M., Liu, Z. T., Salama, A. S., Dong, F., \& Hirota, K. (2015). Bloch sphere-based representation for quantum emotion space. Journal of Advanced Computational Intelligence and Intelligent Informatics, 19(1), 134-142.

Yik, M., Russell, J. A., \& Steiger, J. H. (2011). A 12-point circumplex structure of core affect. Emotion, 11(4), 705-731. Retrieved from http://doi .apa.org/getdoi.cfm?doi=10.1037/a0023980

Yik, M. S. M., Russell, J. A., \& Barrett, L. F. (1999). Structure of self-reported current affect: Integration and beyond. Journal of Personality and Social Psychology, 77(3), 600-619. Retrieved from http://doi.apa.org/getdoi.cfm?doi=10.1037/0022-3514.77.3.600

Yukalov, V. I., \& Sornette, D. (2010). Mathematical structure of quantum decision theory. Advances in Complex Systems, 13(05), 659-698. Retrieved from https://www . worldscientific.com/doi/abs/10.1142/S0219525910002803

Zajonc, R. B. (1980). Feeling and thinking: Preferences need no inferences. American Psychologist, 35(2), 151-175.

Zipf, G. K. (1945). The Repetition of Words, Time-Perspective, and Semantic Balance. The Journal of General Psychology, 32(1), 127-148. Retrieved from http: //www.tandfonline.com/doi/abs/10.1080/00221309.1945.10544486

Řeháček, J., Englert, B.-G., \& Kaszlikowski, D. (2004, nov). Minimal qubit tomography. Physical Review A, 70(5), 052321. Retrieved from https://link.aps.org/doi/10.1103/PhysRevA.70.052321 